ARTICLE

\title{
Hsf1 and Hsp90 orchestrate temperature-dependent global transcriptional remodelling and chromatin architecture in Candida albicans
}

Michelle D. Leach ${ }^{1,2}$, Rhys A. Farrer ${ }^{3}$, Kaeling Tan ${ }^{4}$, Zhengqiang Miao ${ }^{4}$, Louise A. Walker ${ }^{5}$, Christina A. Cuomo ${ }^{3}$, Robert T. Wheeler ${ }^{5}$, Alistair J.P. Brown ${ }^{1}$, Koon Ho Wong ${ }^{4} \&$ Leah E. Cowen ${ }^{2}$

Fever is a universal response to infection, and opportunistic pathogens such as Candida albicans have evolved complex circuitry to sense and respond to heat. Here we harness RNA-seq and ChIP-seq to discover that the heat shock transcription factor, Hsf1, binds distinct motifs in nucleosome-depleted promoter regions to regulate heat shock genes and genes involved in virulence in C. albicans. Consequently, heat shock increases C. albicans host cell adhesion, damage and virulence. Hsf1 activation depends upon the molecular chaperone Hsp90 under basal and heat shock conditions, but the effects are opposite and in part controlled at the level of Hsf1 expression and DNA binding. Finally, we demonstrate that Hsp90 regulates global transcription programs by modulating nucleosome levels at promoters of stress-responsive genes. Thus, we describe a mechanism by which $\mathrm{C}$. albicans responds to temperature via Hsf1 and Hsp90 to orchestrate gene expression and chromatin architecture, thereby enabling thermal adaptation and virulence.

\footnotetext{
${ }^{1}$ Aberdeen Fungal Group, University of Aberdeen, Institute of Medical Sciences, Foresterhill, Aberdeen AB25 2ZD, UK. ${ }^{2}$ Department of Molecular Genetics, University of Toronto, Toronto, Ontario, Canada M5S 1A8. ${ }^{3}$ Genome Sequencing and Analysis Program, Broad Institute of MIT and Harvard, Cambridge, Massachusetts 02142, USA. ${ }^{4}$ Faculty of Health Sciences, University of Macau, Macau SAR 999078, China. ${ }^{5}$ Department of Molecular and Biomedical Sciences, University of Maine, Orono, Maine 04469, USA. Correspondence and requests for materials should be addressed to L.E.C. (email: leah.cowen@utoronto.ca).
} 
C ompared with the environmental extremes encountered by saprobes in nature, mammalian hosts provide relatively stable niches for commensal microorganisms and pathogens. Nevertheless, microorganisms that colonize mammals are continually challenged with a myriad of environmental stimuli such as temperature fluctuations, osmotic imbalances, oxidative and weak-acid stresses, as well as nutrient limitation ${ }^{1}$. Survival, colonization and infection depend upon activation of environmental response pathways that have been fine-tuned over evolutionary time to drive physiological adaptation ${ }^{2,3}$.

The potential for optimization of circuitry governing sensing and responding to host conditions is greatest for microbes that are natural members of commensal microbiomes. This includes the diverse bacteria that promote host immune function and health, as well as fungi that have only recently been appreciated to play important roles in defining commensal communities ${ }^{4}$. Commensal fungi are capable of becoming opportunistic pathogens that can cause life-threatening infections. One such fungus is Candida albicans, which has evolved as a relatively harmless commensal of the mucous membranes and digestive tracts of healthy individuals. When host antimicrobial defences are compromised, this yeast can cause superficial mucosal infections in otherwise healthy individuals ${ }^{5}$, and up to 400,000 life-threatening systemic infections in immunocompromised patients per year6.

With fever as a ubiquitous host response to infection, it follows suit that the capacity to sense and respond to thermal cues is one of the most important and conserved stress responses in nature. The heat shock response is characterized by global pausing of translation elongation ${ }^{7}$ and remodelling of gene expression programs distinguished by the induction of heat shock proteins (HSPs), such as chaperones that aid in protein folding ${ }^{3}$. The master regulator of this transcriptional response is the heat shock transcription factor (HSF), which is conserved from yeasts to humans ${ }^{8,9}$. Hsf1 is essential for viability in C. albicans and other yeasts, likely due to its function in enabling core gene expression programs ${ }^{10,11}$. HSF binds to the major groove of cis-acting DNA sequence motifs termed heat shock elements (HSE), characterized by tandem inverted repeats of the consensus sequence $5^{\prime}$-nGAAn-3' (refs 12,13). In Saccharomyces cerevisiae, transcription of $\sim 10 \%$ of genes is modulated in response to heat shock $^{3}$, and nearly $3 \%$ of genes are directly bound by Hsf1 (ref. 14). Transcription initiation in response to elevated temperature is also influenced by nucleosome positioning, with heat shock resulting in increased nucleosome occupancy at repressed promoters and decreased occupancy at active promoters $^{15}$.

Cellular adaptation to thermal stress is modulated by complex functional relationships between Hsf1 and the molecular chaperone Hsp90, which influence signalling and gene expression. As with many chaperones, Hsfl enables basal expression and temperature-dependent activation of Hsp90-encoding genes ${ }^{16}$. Hsp90 controls the Hsf1-HSE regulon through an autoregulatory circuit ${ }^{17,18}$, activation of which is essential for virulence of C. albicans ${ }^{19}$. Beyond Hsf1, Hsp90 stabilizes diverse cellular regulators ${ }^{20}$, and exerts additional control on gene expression. In Drosophila, Hsp90 targets paused RNA polymerase II (ref. 21) and in S. cerevisiae, deletion of HSC82 (the constitutively expressed HSP90 isoform) delays nucleosome removal from the GAL1 promoter $^{22}$. Despite the profound impact of temperature-dependent regulation of Hsfl and Hsp90 on virulence traits in fungal pathogens ${ }^{23-25}$, the mechanisms by which these global regulators orchestrate temperature-dependent signalling, gene expression and virulence has remained elusive. Here, we use genome-scale approaches to explore how $C$. albicans responds to thermal insults, revealing that Hsf1 and Hsp90 govern nucleosome positioning, gene expression and virulence traits.

\section{Results}

Hsf1 binds genes required for stress adaption and virulence. Using chromatin immunoprecipitation (ChIP) of TAP-tagged Hsf1 (Hsf1-TAP) followed by sequencing (ChIP-seq), we set out to identify the genome-wide direct targets of Hsfl in the absence $\left(30^{\circ} \mathrm{C}\right)$ or presence of heat shock $\left(30-42^{\circ} \mathrm{C}\right)$. Distinct Hsf1-TAP ChIP-seq signals were identified at 49 genomic sites in the absence of heat shock. Upon heat shock, 104 Hsf1-binding sites were identified, including all 49 observed in the absence of heat stress (Fig. 1 and Supplementary Data 1). We hereafter refer to the 49 sites as constitutive targets, and the remaining 55 sites as heat shock-dependent targets. The majority of binding events were found at promoter regions close to transcription start sites (Supplementary Fig. 1a), with transcription of genes closest to Hsf1-binding sites being highly upregulated upon heat shock (Supplementary Fig. 1b). Our results suggest that Hsf1 constitutively binds to a set of target promoters before heat shock pending activation.

Many Hsf1 target genes identified in our study encode proteins implicated in stress responses such as molecular chaperones, oxidative stress regulators, and ubiquitination and proteolysis factors. Unexpectedly, Hsfl regulates its own expression and also bound upstream of genes required for adhesion, filamentous growth and pathogenesis (Fig. 1b,c and Supplementary Data 2). GO term analysis of Hsfl-bound genes revealed enrichment of the categories 'protein-folding' (hypergeometric distribution $P=1.87 \mathrm{e}-20)$ 'response to heat' (hypergeometric distribution $P=0.00074)$ and 'entry into the host' (hypergeometric distribution $P=0.0052$ ) (Supplementary Data 3), suggesting that Hsf1 is not only required for regulating HSPs in response to heat shock but also genes involved in virulence.

Metagene analysis was performed to compare the regulation of Hsf1 target genes with constitutive and heat-induced binding at their promoters in response to heat shock. Transcription (RNA polymerase (Pol) II occupancy measured by Pol II ChIP-seq) of genes with constitutive Hsfl binding increased dramatically in response to heat shock (Supplementary Fig. 1c). This is accompanied by a drastic increase in Hsfl-binding levels (Supplementary Fig. 1d), suggesting that Hsf1 is critical for their upregulation during thermal insults. In contrast, heat-induced Hsf1 targets display lower activation in response to heat shock (Supplementary Fig. 1c), with consistently lower levels of Hsfl binding at these promoters (Supplementary Fig. 1d). Taken together, these observations suggest that the magnitude of Hsf1-dependent activation during heat shock is contingent upon its level of binding before heat shock.

C. albicans Hsf1 target genes may also be regulated by additional transcription co-factors. For example, ALS3 is coregulated by $\mathrm{Nrg} 1$ and Tup1 (ref. 26), the ALS1 promoter is bound by Bcr1, Tec1, Efg1, Ndt80 and Brg1, and the ROB1 promoter is bound by Tec1, Efg1, Ndt80 and Rob1 (ref. 27). Therefore, we tested whether upregulation of these Hsf1 targets in response to heat shock is contingent upon Hsf1. To achieve this, we used a tetO-HSF1/hsf1D strain to deplete HSF1 and monitored the expression of genes required for thermal adaption (HSP90, HSP104 and $H S P 21)^{28}$, adhesion and virulence (ALS1 and ALS3) $)^{29,30}$ and biofilm formation $(R O B 1)^{27}$ (Supplementary Fig. 2). Depletion of HSF1 abrogated the heat shock-dependent upregulation of key HSPs: HSP90, HSP104 and HSP21. Further, we found that $A L S 3, A L S 1$ and $R O B 1$ expression increased significantly in response to heat shock, and that depletion of HSF1 reduced this upregulation (Supplementary Fig. 2). 
a

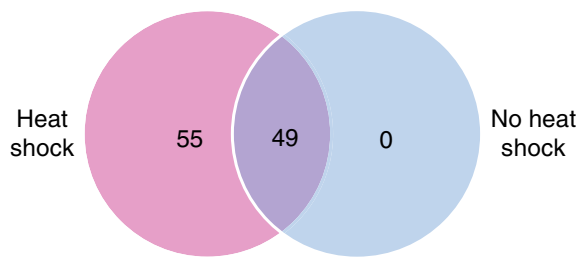

b

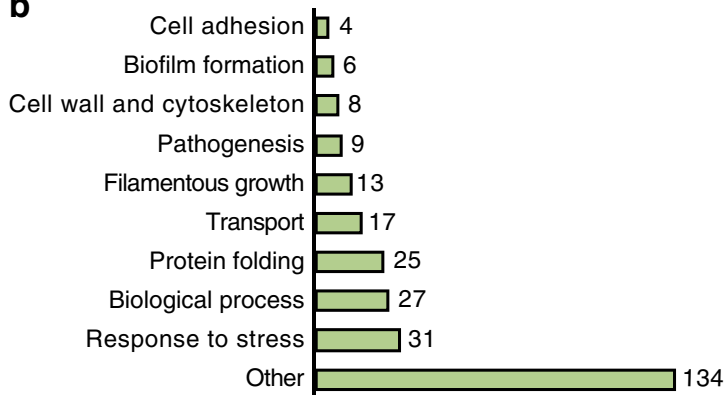
134
C

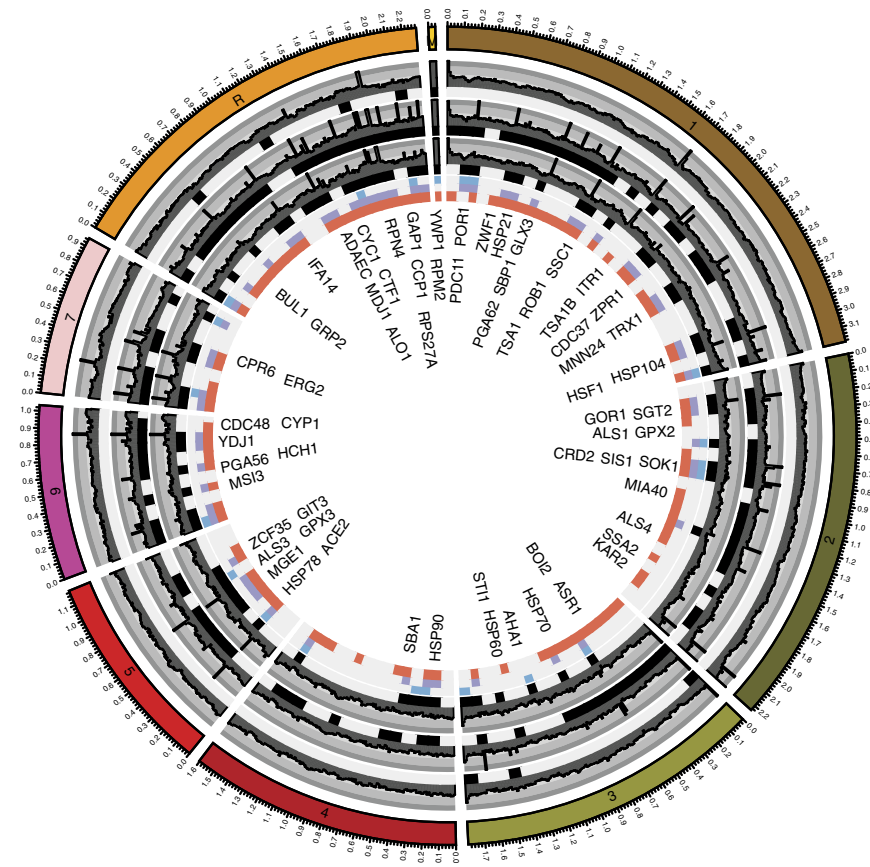

Figure 1 | Hsf1 target genes. (a) Venn diagram depicting number of Hsf1 peaks in the absence (no heat shock, blue) or presence (heat shock, pink) of a $30-42{ }^{\circ} \mathrm{C}$ heat shock. (b) GO Slim Mapper analysis was performed on the ChIP-seq data set to identify the processes of all genes bound by Hsf1 in the absence and presence of a $30-42^{\circ} \mathrm{C}$ heat shock. Number of $\mathrm{Hsf} 1$ target genes represented under each category are indicated. Other categories include, but are not limited to, response to drug, cell cycle, response to chemical, organelle localization, protein catabolic process, translation, ribosome biogenesis, signal transduction and cell development. (c) Circos plot illustrating peaks of genes bound by Hsf1 (systematic names) in the absence and presence of a $30-42{ }^{\circ} \mathrm{C}$ heat shock. Each circle from the periphery to the core represents the following: chromosomal location; normalized read depth of peaks from untagged samples, heat shocked samples and untreated samples; key of condition under which the peak is present (untreated, untreated with heat shock and heat shock only); and name of gene bound. All boxes represent $\geq 1$ peak that falls within a $100 \mathrm{~kb}$ non-overlapping window.

Thus, Hsf1 regulates the expression of not only heat shock genes but also key virulence genes in response to heat shock.

Hsfl binds canonical and non-canonical heat shock elements. The findings that there are two classes of Hsf1-binding targets (constitutive and heat-induced), and that Hsf1 activation level is correlated with its binding pattern prompted us to search for enriched motif(s) at the promoters of Hsfl targets. We began with de novo motif discovery on the full set of Hsf1-binding sites (200 bp spanning the summit of $104 \mathrm{Hsfl-TAP}$ ChIP-seq peaks) and identified one highly significant consensus sequence $(E$-value $=1.5 \mathrm{e}-164) \quad$ (Fig. 2a). This motif, conforming to a pattern of three inverted nGAAn repeats, is similar to both the human $^{31}$ and S. cerevisiae ${ }^{14} \mathrm{Hsfl}$ recognition motifs, suggesting that $C$. albicans Hsf1 probably functions as a trimeric complex as with other HSF homologues ${ }^{8}$. However, only 29 out of the 104 Hsfl ChIP-seq peaks contained the consensus sequence TTCnnGAAnnTTC (Fig. 2b), indicating that Hsf1 likely recognises additional motif(s) in C. albicans.

We extended our analysis to search for additional sequence element(s). Using de novo motif discovery and manual inspection, we found that the motifs GAAnnTTC and TTCn 7 TTC (25 and 55 out of 104, respectively) are also enriched among Hsf1-bound regions (Fig. 2b). The three motifs identified are found within $100 \mathrm{bp}$ of the centre of Hsf1-TAP ChIP-seq peaks with strong ChIP-seq signals (Supplementary Fig. 3a and Supplementary Data 4), indicating they are probable Hsf1-binding elements. The latter motif is a shorter version of the step-type non-canonical HSE (nTTCn TTCn $_{7}$ TTC) identified in S. cerevisiae ${ }^{32}$, while the former motif has not been reported for Hsf1 in other organisms. It is the most predominant motif among the three, present at a higher frequency among Hsfl-bound peaks than the $S$. cerevisiae step-type non-canonical motif (Fig. 2b). These findings suggest that $C$. albicans and $S$. cerevisiae Hsfl have related yet distinct binding affinities, and that C. albicans Hsf1 is capable of binding to nGAAn elements arranged in at least three configurations, presumably in different dimeric and trimeric forms.

A further 4,879 genes with the Hsfl consensus sequences present in their promoter were identified, but no detectable Hsf1 binding was observed under our ChIP-seq experimental conditions (Fig. 2c and Supplementary Data 4). These genes were enriched for functions related to transport, stress responses, 

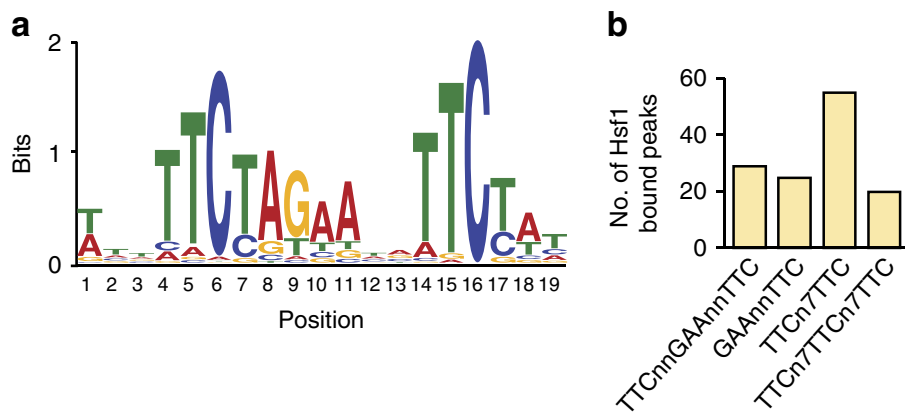

$$
\begin{array}{ll}
\text { C } \quad \square \text { Hsf1 bound } \\
\square \text { Non-Hsf1 bound }
\end{array}
$$

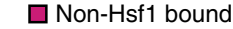

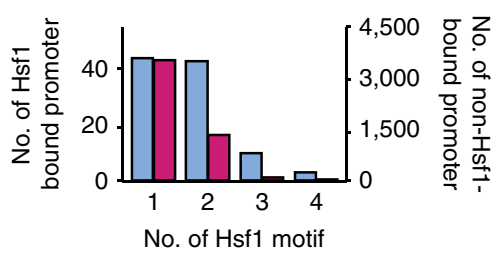

No. of Hsf1 motif
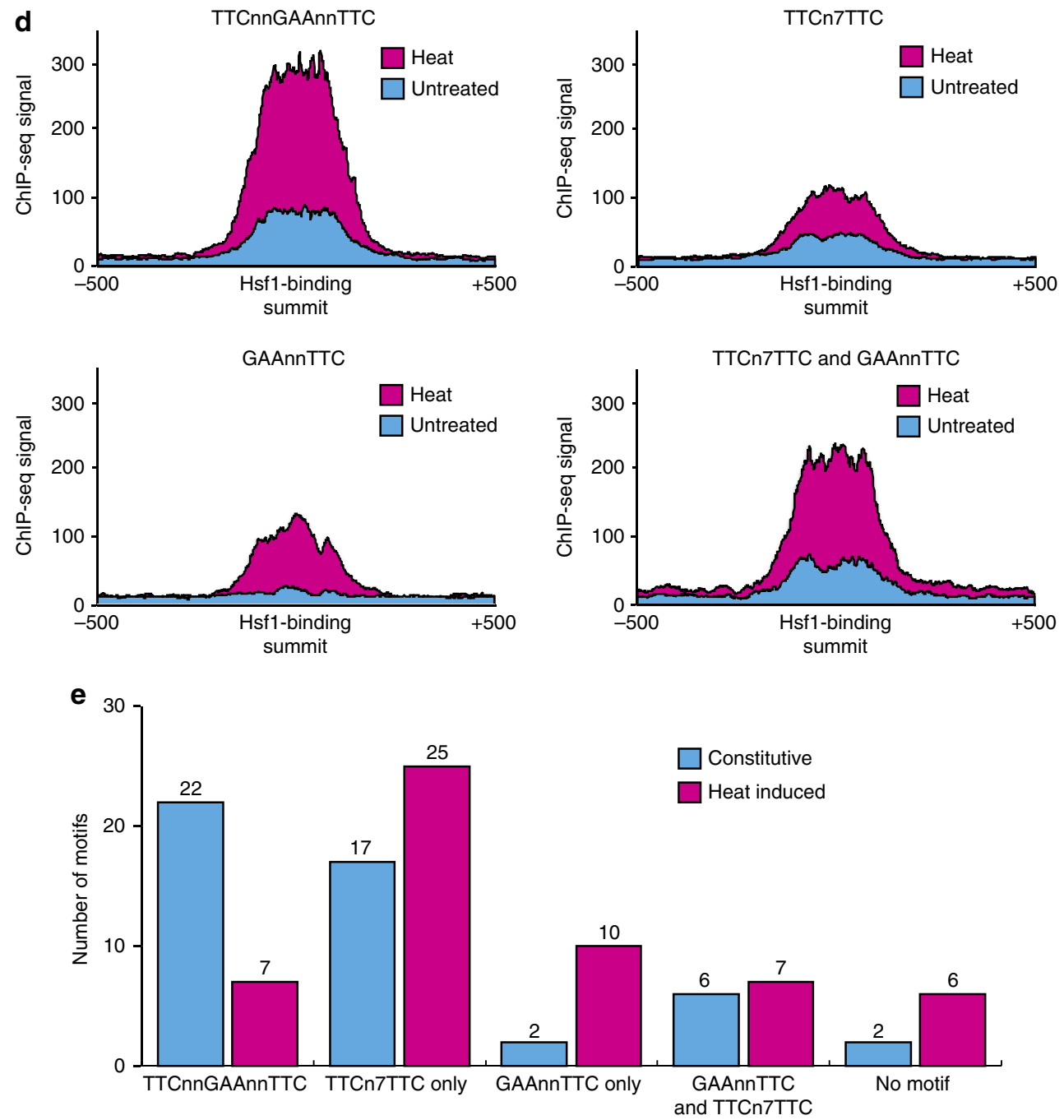

Hsf1 motif types

Figure 2 | Hsf1-binding motifs. (a) De novo motif analysis using MEME on Hsf1-binding sites identified this highly significant sequence $(E$-value $=1.5 e-164)$. (b) Bar graph representing the number of Hsf1 targets containing one of four motifs identified using de novo motif discovery and manual inspection. (c) Histogram illustrating the number of different Hsf1 motifs found in Hsf1-bound (blue) versus non-Hsf1-bound promoters (red). (d) Hsfl binding signal as measured by Hsf1 ChIP-seq at promoters containing the indicated Hsf1 motifs under untreated (blue) and heat shock (red) conditions. (e) Bar graph illustrating number of Hsf1-binding events with the indicated motifs. Constitutively bound Hsf1 genes are represented in blue, heat induced Hsf1 genes are represented in red.

filamentous growth, cell cycle, translation and metabolism, and may be bound by Hsfl in response to specific cues. Our analysis also highlighted that the TTCnnGAAnnTTC and TTCn7TTCn7TTC motifs are highly enriched among Hsfl-bound promoters compared with promoters that have Hsf1 motifs without Hsfl binding (Supplementary Fig. 3b). Moreover, the majority of Hsfl-bound promoters contain at least one type of Hsf1 motif (Supplementary Fig. 3c). We also discovered that many Hsf1 targets contain multiple Hsf1 motifs (for example, GAAnnTTC repeats $1 \mathrm{~kb}$ upstream of the ATG), with two promoters (TSA1B and HSP70) having as many as nine motifs (Supplementary Fig. 3c), suggesting a strong selection pressure for $\mathrm{Hsfl}$ regulation of these target genes. 
Hsf1 binds to the three identified motifs with different binding affinities. The TTCnnGAAnnTTC element is most strongly bound, while GAAnnTTC and TTCn7TTC have a reduced but still significant binding affinity (Fig. 2d). The TTCnnGAAnnTTC element was enriched among promoters with constitutive Hsf1 binding, whereas heat-induced Hsfl-binding events were associated with the other two motifs with weaker Hsf1-binding affinity (Fig. 2e). Consistently, the level of Hsfl bound at heatinduced promoters was lower than the level bound at constitutive promoters in both the absence and presence of heat shock (Supplementary Fig. 1d). Together, our results suggest that the differential binding affinity of Hsfl in the absence and presence of heat is associated with the configuration of nGAAn elements at target promoters.

Nucleosome positioning regulates Hsf1 binding to DNA. Nucleosomes have a profound impact on the accessibility of DNA to transcription factors and on transcription initiation ${ }^{15}$, thus we sought to determine if nucleosomes affect Hsf1 DNA binding. At genomic regions with Hsfl binding, nucleosomes (measured by histone H3 ChIP-seq) were depleted near Hsf1 motifs (Fig. 3a). As a control, we examined nucleosome density at 104 random genomic regions, finding that nucleosome depletion is specific to Hsf1-bound regions (Fig. 3b). Further, we analysed genomic regions containing an $\mathrm{Hsfl}$ consensus sequence that were not bound by Hsf1 in our experimental conditions, and found that nucleosome density was consistently high across these recognition motifs (Fig. 3c). Together, this suggests that nucleosome positioning at Hsfl motifs influences the ability of Hsf1 to recognize and bind DNA.
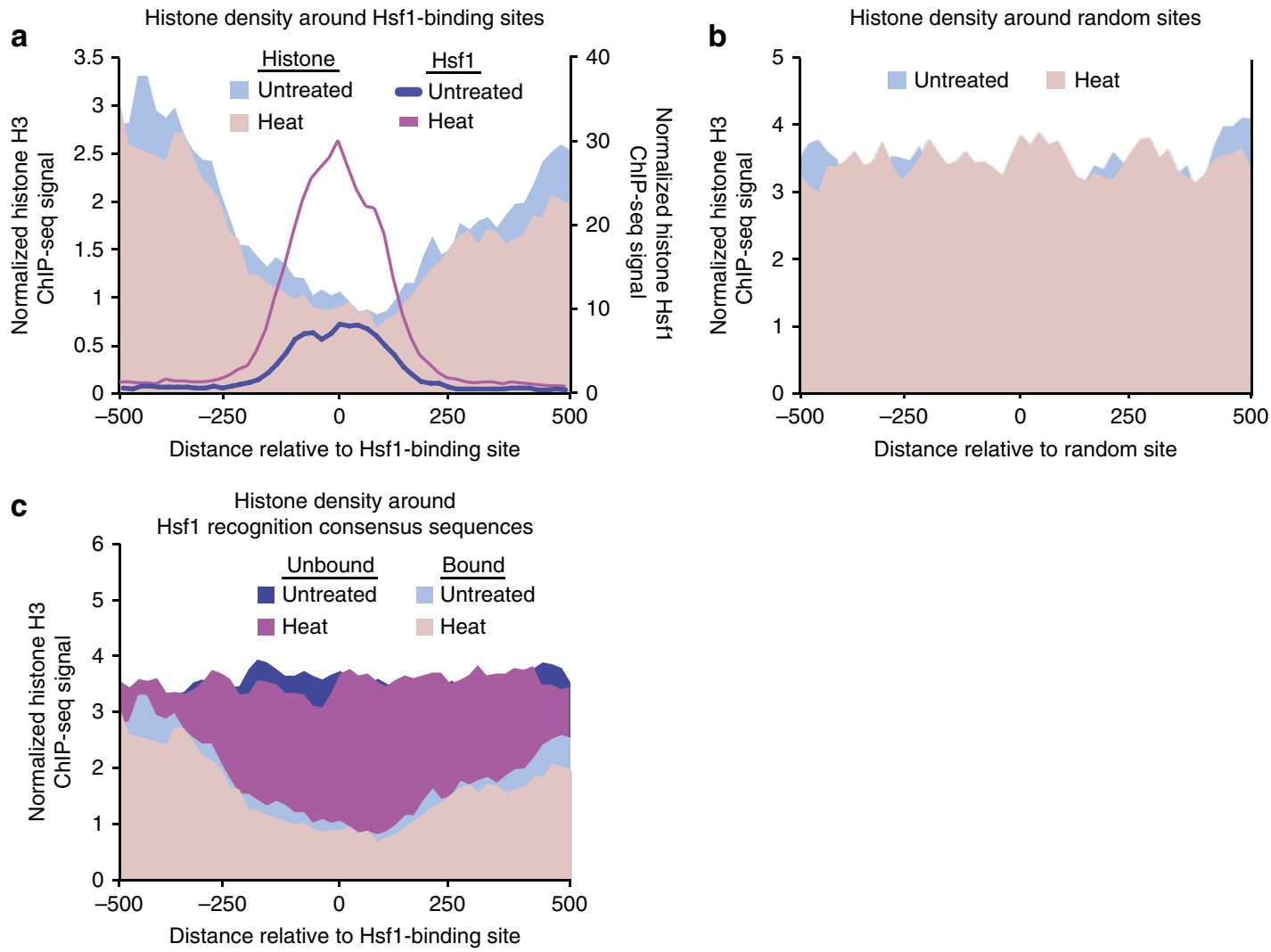

This result prompted us to investigate whether the different binding affinities at the three Hsfl binding motifs were also mediated through nucleosome density, but no obvious correlation was found (Supplementary Fig. 4a). Specifically, nucleosome levels at both Hsf1-binding sites (Fig. 3a) and Hsf1-regulated promoters (1 kb upstream of ATG) (Supplementary Fig. 4b) did not significantly differ upon heat shock, suggesting that Hsf1 activation and heat shock do not invoke significant nucleosome eviction at these heat shock-induced promoters.

Heat shock increases virulence of $C$. albicans in vivo. Our data demonstrate that Hsfl binds to genes involved in virulence, adhesion and biofilms during heat shock, but these genes may play an as yet undetermined role in thermal adaptation. To assess this, als $3 \Delta /$ als $3 \Delta$ and rob $1 \Delta /$ rob $1 \Delta$ mutants, along with their wild-type counterparts, were heat shocked and cell viability was determined by colony forming units (CFUs). The mutants displayed no significant heat shock sensitivity compared with their respective wild-type controls (Supplementary Fig. 5a). Thus, these genes are not required for thermal adaptation.

C. albicans has evolved in endothermic animals as an opportunistic pathogen. Thus we postulated that it exploits thermal insults to upregulate its virulence program via Hsf1. Further, our previous studies established that a prior heat shock protects cells from oxidative stress, which could enable C. albicans to resist killing by the host immune response ${ }^{18}$. To test the impact of heat shock on virulence traits, we utilized the TR146 oral epithelial carcinoma cell lines as a well-established model to investigate adhesion and cell damage ${ }^{33,34}$. Cells primed with heat shock displayed $15 \%$ greater adherence than cells grown 
at $30^{\circ} \mathrm{C}$ alone (Student's $t$-test $P<0.05$ ) (Fig. 4a). This is likely due to the Hsf1-dependent upregulation of $A L S$ genes, which are effectors of epithelial adhesion and invasion ${ }^{35,36}$. Next, we assayed epithelial cell damage by measuring the release of lactate dehydrogenase (LDH) from TR146 cells. After $20 \mathrm{~h}$ co-incubation, damage increased when $C$. albicans had received a prior heat shock $(P<0.05)$ (Fig. $4 \mathrm{~b})$. These results cannot be attributed to morphological changes during heat shock, as cells remain in the yeast form up to $120 \mathrm{~min}$ post $30-42^{\circ} \mathrm{C}$ heat shock $^{18}$. Our data suggest that a drastic heat shock activates a C. albicans program associated with increased host cell adhesion and damage.

To evaluate the effect of heat shock on virulence, we utilized two complementary infection models, the greater wax moth (Galleria mellonella) and zebrafish (Danio rerio). G. mellonella larvae infected with heat shocked $C$. albicans exhibited increased mortality rates compared with controls $(P<0.05$, Log-rank test $)$ (Fig. 4c). These results were recapitulated with the zebrafish infection model, with increased mortality of zebrafish infected with $C$. albicans cells that had received a prior heat shock compared with controls $(P<0.05$, Log-rank test) (Fig. $4 \mathrm{~d})$. Thus, an acute heat shock increases virulence in two models of Candida infection.

We further corroborated our results using a temperature shift that more closely recapitulates physiological conditions associated with a febrile host. C. albicans has been isolated from skin, which is typically $33^{\circ} \mathrm{C}$; it can then become internalized, and subsequently local inflammation or fever can raise temperatures as high as $41^{\circ} \mathrm{C}$. We previously established that Hsfl initiates the classic heat shock response during slow thermal transitions that would mimic fever, but also during sharp transitions from $37-42^{\circ} \mathrm{C}$ (ref. 37). To determine if different temperature shifts have comparable effects on virulence traits, we repeated the TR146 adhesion assay using a $37-42^{\circ} \mathrm{C}$ heat shock, and found that adhesion increased in cells that had received a prior heat shock, albeit not to the same extent as cells that received a $30-42{ }^{\circ} \mathrm{C}$ heat shock (Supplementary Fig. 5b). Further, the impact of Hsf1 on expression of key genes for heat shock (HSP90, HSP104 and HSP21) and virulence (ALS1, ALS3, ACE2 and $R O B 1$ ) was also maintained in response to a $37-42^{\circ} \mathrm{C}$ heat shock, though not to the same magnitude as with the $30-42{ }^{\circ} \mathrm{C}$ heat shock. (Supplementary Fig. 5c). Therefore, our data suggest that Hsf1 coordinates cellular responses to host-relevant thermal shifts.

Hsp90 remodels global transcription during heat shock. The emergent paradigm from decades of research has been that Hsp90 physically interacts with Hsfl to repress its activating function, such that when Hsp90 functional capacity is overwhelmed by elevated temperature or other perturbations, the repression is relieved and Hsfl activates the heat shock response ${ }^{18}$. However, recent studies have implicated $\mathrm{Hsp} 90$ in directly regulating gene expression through chromatin remodelling, RNA polymerase II pausing and nucleosome removal at target promoters ${ }^{21,22,38}$. To assess the impact of Hsp90 on the transcriptional response to heat shock, we performed RNA-seq in a wild-type and tetO-HSP90/hsp904 strain at 10, 30 and $60 \mathrm{~min}$ post heat shock. In wild-type cells, heat shock elicited rapid, large-scale remodelling of gene expression. At $10 \mathrm{~min}$ post heat shock,
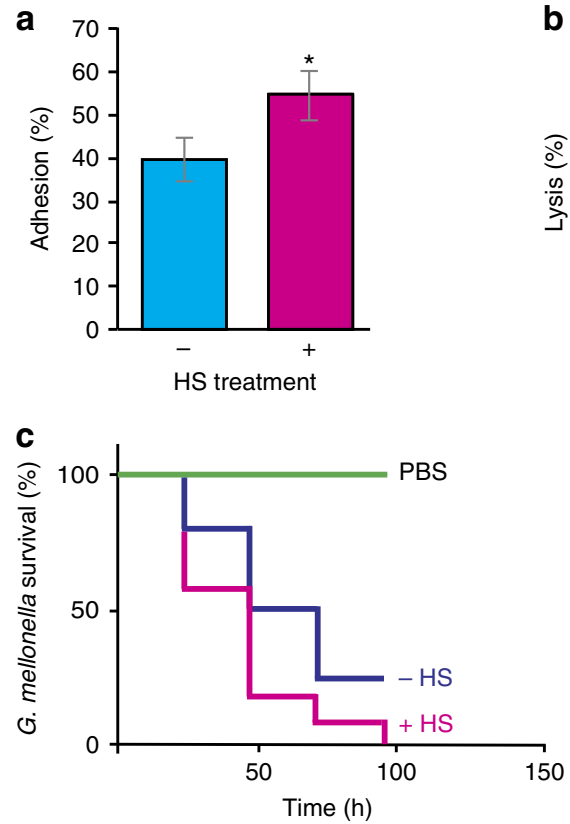

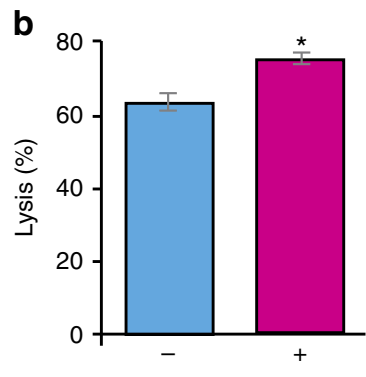

HS treatment

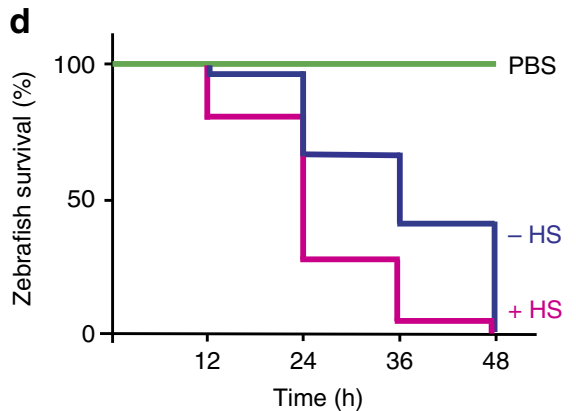

Figure 4 | Heat shock increases pathogenicity traits. (a) Increased adhesion of C. albicans wild-type (SN95) cells to TR146 epithelial cells post $30-42^{\circ} \mathrm{C}$ HS. Results represent percentage of adhered cells from $30^{\circ} \mathrm{C}$ grown cells (no HS) or $30-42^{\circ} \mathrm{C}$ heat-shocked cells (HS) compared with control CFUs. Data represent mean values \pm s.e.m. from two independent biological replicates. Student $t$-test, ${ }^{\star} P<0.05$ compared with wild type. (b) Increased cell damage by $\mathrm{C}$. albicans wild-type (SN95) cells post $30-42^{\circ} \mathrm{C}$ HS to TR146 epithelial cells. Culture medium was assessed for LDH release as a measure of epithelial cell damage of TR146 cells after incubation with C. albicans grown at $30^{\circ} \mathrm{C}$ (no HS) or subjected to a 15 min $30-42^{\circ} \mathrm{C} \mathrm{HS}$. Data represent mean values \pm s.e.m. from two independent biological replicates. Student $\mathrm{t}$-test, ${ }^{\star} P<0.05$ compared with the wild type. (c) HS increases virulence in the G. mellonella model of infection. Twenty larvae per treatment group were injected with $2.5 \times 10^{5}$ cells of $\mathrm{C}$. albicans wild-type (SN95) cells grown at $30{ }^{\circ} \mathrm{C}$ (no HS) or subjected to a $15 \mathrm{~min} 30-42^{\circ} \mathrm{C} \mathrm{HS}$. PBS was used as a control. Data are representative of four biological replicates. Significance was determined using Log-rank (Mantel-Cox) test, with a $P<0.001$ (d) HS increases C. albicans virulence in the zebrafish model of infection. Ninety zebrafish larvae per treatment group were injected with $15-20 \mathrm{C}$. albicans wild-type (SN95) cells grown at $30^{\circ} \mathrm{C}$ (no HS), wild-type cells subjected to a 15 min $30-42{ }^{\circ} \mathrm{C}$ HS or with a PBS control. Significance was determined using Log-rank (Mantel-Cox) test, with a $P<0.0001$. HS, heat shock. 
766 genes were $>2$-fold upregulated, increasing to 1,119 genes by $60 \mathrm{~min}$, representing $\sim 18 \%$ of $C$. albicans genome (Supplementary Fig. 6a,b and Supplementary Data 5). GO term analysis across the three time points supported the hypothesis that a primary effect of heat shock is protein unfolding (Supplementary Fig. 6c), as well as modulation of genes involved in the proteasome/ubiquitination and oxidative stress response. Notably, C. albicans also upregulated genes related to cell cycle, biofilm formation and pathogenesis, in keeping with our findings that an acute heat shock increases pathogenesis (Fig. 4). Heat shock also downregulated a large portion of protein-encoding genes in $C$. albicans, enriched in different metabolic processes and ribosome biogenesis (Supplementary Fig. 6a).

Hsp90 had a profound impact on the transcriptional response to heat shock. Upon HSP90 depletion, 10 min post-heat shock, only 358 of 6,197 genes were $>2$-fold upregulated, compared with 766 in wild-type cells, and of these, only 228 were common to the wild-type program (Fig. 5a,b). These genes were enriched for protein folding and refolding, similar to wild-type cells (Supplementary Table 1). Advancing to 30 and $60 \mathrm{~min}$ post treatment, the number of genes upregulated $>2$-fold increased to 1,056. However, these genes were enriched for cytoskeleton organization, nuclear division, chromosome segregation and cell cycle-related processes, reinforcing functional connections between Hsp90 and cell cycle control at high temperature in $S$. cerevisiae ${ }^{39}$. By $60 \mathrm{~min}$, an enrichment of ERAD (endoplasmic-reticulum-associated protein degradation) pathway genes were found, while protein-folding categories were no longer enriched, suggesting that the combined stress is so pernicious that cellular resources are diverted to targeting unfolded proteins for degradation. Notably, depleting HSP90 in the absence of any stress caused upregulation of 515 genes by $>2$-fold (Supplementary Data 5). GO term analysis reveals an enrichment of genes involved in metabolic processes (Supplementary Data 6), but no enrichment of genes involved in general stress responses. This suggests the transcriptional effects observed in response to HSP90 depletion are not due to non-specific stress from mis-folded proteins. Metabolic and ribosome biogenesis genes remained downregulated upon depletion of HSP90 during heat shock (Fig. 5c). Therefore, Hsp90 is required for normal upregulation of most heat shock inducible genes during heat shock.

A strong temporal signature underlies the impact of HSP90 depletion. Expression of over $75 \%$ of Hsf1 target genes was drastically affected upon HSP90 depletion after a 10 min heat shock (Fig. 5d), and the observed effect is mainly due to the loss of transcriptional induction upon heat shock (Supplementary Fig. 7a). However, changes in expression of Hsfl targets between wild-type and HSP90-depleted cells gradually became less pronounced post heat shock (Supplementary Fig. 7b). Thus, Hsp90 is crucial for rapid mobilization of the heat shock response.

Hsp90 modulates Hsf1 DNA binding and expression. Hsp90 is known to interact with $\mathrm{Hsfl}$ in $\mathrm{C}$. albicans, inhibiting its full activation $^{18}$. We therefore expected Hsfl binding to increase upon HSP90 depletion. Indeed, overall Hsfl-TAP ChIP-seq signals at Hsfl targets increased upon HSP9O depletion compared with wild-type (Fig. 6a). Moreover, $18 \%$ of Hsf1 targets were upregulated $>1.5$-fold upon HSP9O depletion in the absence of heat shock, supporting the established model that Hsp90 represses Hsfl function in the absence of heat shock. These genes were enriched for GO categories including response to heat, protein unfolding and heat acclimation (Supplementary Table 2). In contrast, Hsfl binding at target promoters $15 \mathrm{~min}$ post heat shock was markedly reduced upon HSP90 depletion (Fig. 6a), which would account for the reduced expression of Hsf1 target genes during early heat shock.

Before heat shock, HSF1 expression is elevated upon HSP90 depletion (Fig. 6b). This is consistent with the increase in Hsfl DNA binding and Hsf1-dependent gene expression we observed. Strikingly, the induction of HSF1 expression in response to heat shock observed in wild-type cells was abolished 10 min post heat shock upon depletion of HSP90 (Fig. 6c). Thus, under basal conditions, Hsp90 represses Hsf1 such that depletion of HSP90 induces HSF1 expression, binding and induction of genes required for protein re-folding. However, Hsp90 is required for the rapid mobilization of the heat shock response, such that depletion of HSP90 impairs induction of HSF1 leading to a delayed response.

Hsp90 maintains nucleosome-free regions at promoters. Given the striking differences in global gene expression upon heat shock in wild-type versus HSP90-depleted cells, and the discovery that nucleosome positioning modulates Hsf1 binding to DNA, we postulated that Hsp90 may affect gene expression via nucleosome positioning in $C$. albicans. Using histone $\mathrm{H} 3 \mathrm{ChIP}$-seq analysis of wild-type and tetO-HSP90/hsp90D, we found that HSP90 depletion caused nucleosome re-arrangements at Hsf1-regulated promoters under both untreated and heat shock conditions (Fig. 7a,b). Specifically, we observed that nucleosome density at distal promoter regions was reduced. However, nucleosome density increased at the nucleosome-depleted region of Hsf1-regulated genes that overlap with Hsf1-binding sites (Fig. $7 \mathrm{a}, \mathrm{b})$. At the level of individual promoters, 71 and $87 \%$ of Hsf1 regulated promoters showed an increase in histone $\mathrm{H} 3$ levels at the nucleosome-free region upon HSP90 depletion before and after heat shock, respectively (Supplementary Fig. 7c,d). Importantly, the increased histone $\mathrm{H} 3$ levels correlated with the effect of HSP90 depletion on heat induction (as measured in Fig. 5c) (Supplementary Fig. 7e). Taken together, these findings suggest that the effect of Hsp90 on Hsf1 DNA binding could also be mitigated through alterations of the chromatin context at promoters by masking Hsfl DNA-binding motifs.

To assess whether the impact of Hsp90 on chromatin is Hsf1-specific or more global, we extended the analysis to genes with increased (Hsp90 Repressed), decreased (Hsp90 Dependent) and unchanged (Hsp90 Independent) expression as measured by PolII ChIP-seq upon HSP90 depletion, as well as to the RNA polymerase II transcribed tRNA genes as a control (Supplementary Data 7). We identified significant effects on chromatin upon HSP90 depletion only among the category of genes with decreased expression (Fig. 7c). Strikingly, nucleosome positioning was observed primarily at the nucleosome-depleted region but not the distal promoter and gene body of Hsp90-dependent genes (Fig. 7c and Supplementary Fig. 7f). Nucleosome-depleted regions are a critical property of core promoters for transcription factor function and transcription initiation $^{40}$, suggesting that Hsp90 orchestrates gene expression in C. albicans via nucleosome positioning.

Hsp90 may modulate nucleosome positioning by multiple mechanisms. First, one of the 143 genes that are Hsp90-repressed is an ortholog of S. cerevisiae FPR3, orf19.1030. This gene is involved in nucleosome assembly and deposition ${ }^{41}$. Second, the transcription factor, Cbf1, which is involved in nucleosome positioning in $S$. cerevisiae c2 $^{42}$ was repressed upon HSP90 depletion. Third, 8 out of 11 genes known to encode histones are repressed when $H S P 90$ is depleted. These genes were also repressed upon heat shock, indicating that Hsp90 is required for full expression of histone genes, and sequestration of Hsp90 


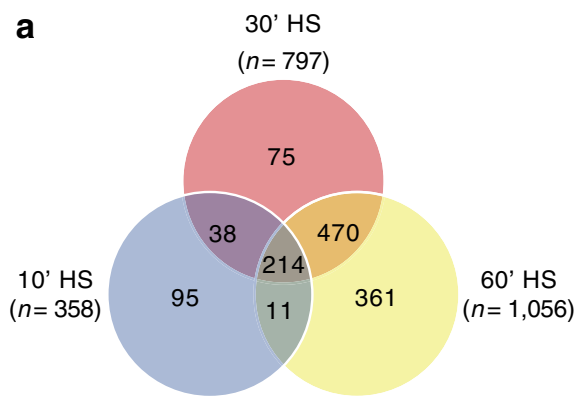
b WT tetO-HSP9O/hsp90 10' HS 10' HS
$(n=766) \quad(n=358)$

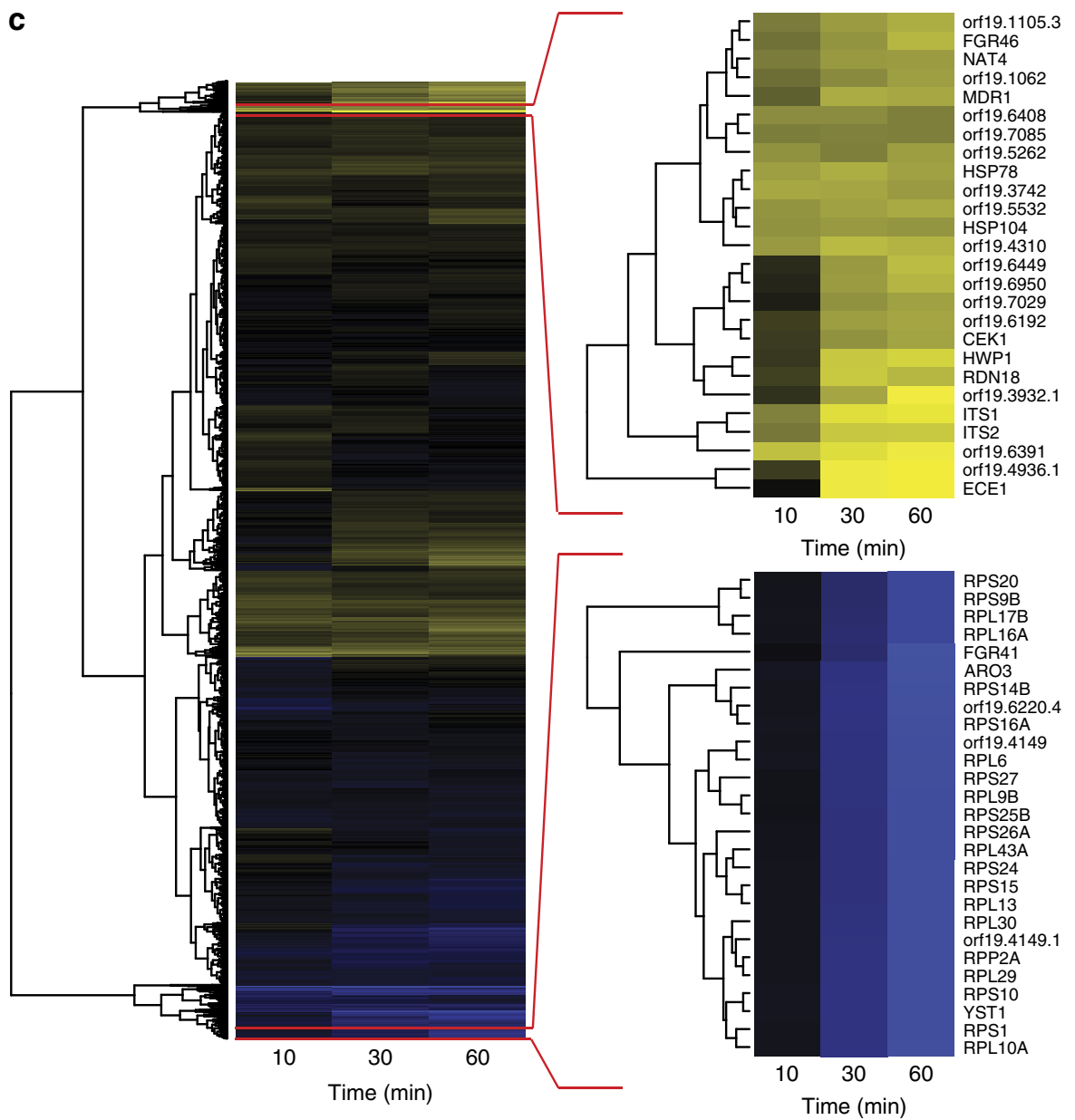

d
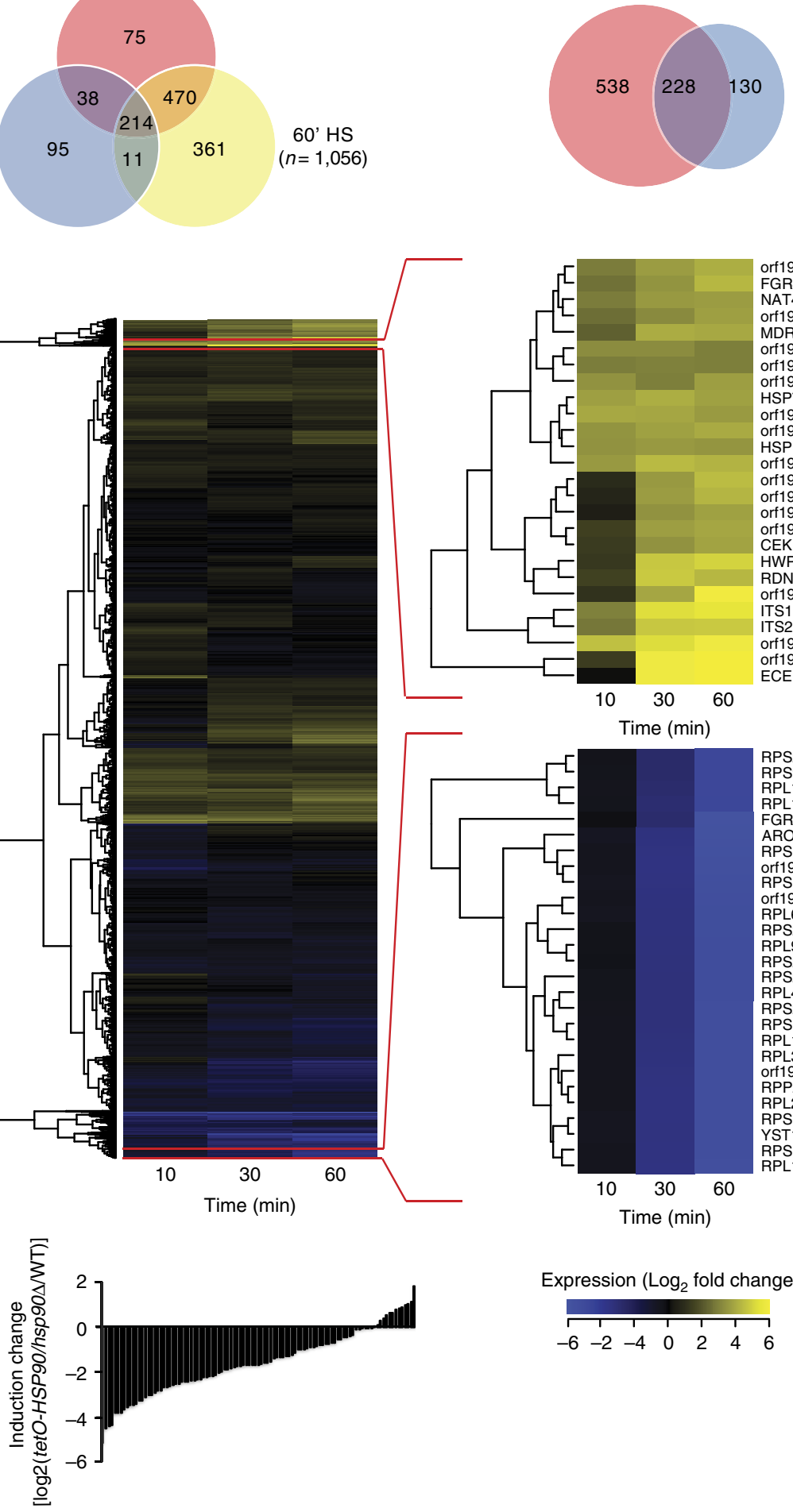

c 
a

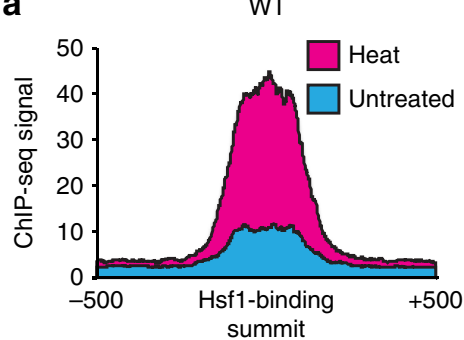

b

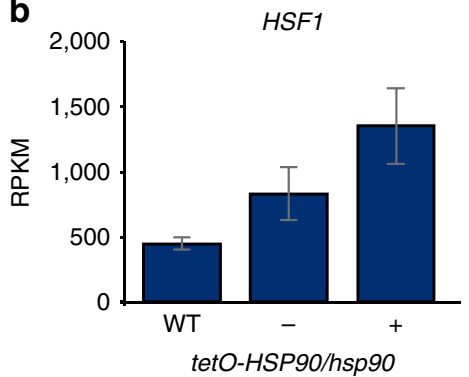

tetO-HSP90/hsp90

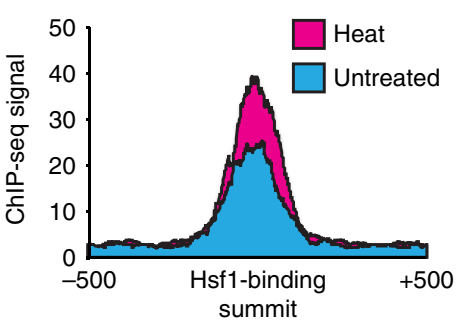

C

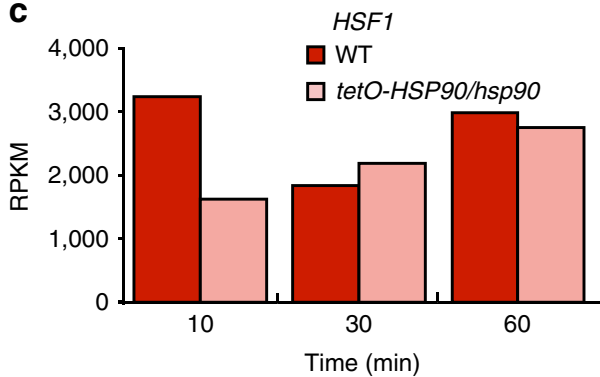

Figure 6 | Hsp90 modulates expression of HSF1. (a) Depletion of HSP9O increases the binding signal of Hsf1 in the absence of heat shock compared with the wild type. Blue represents untreated, red represents heat shocked cells. (b) Expression of HSF1, measured by RNA-seq, increases upon HSP90 depletion

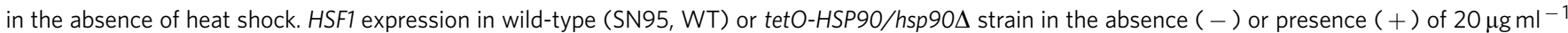
doxycycline to repress HSP90 expression. Data represent mean values \pm s.d. from three independent biological replicates (c) Heat induction of HSF1 expression in wild-type (dark red) versus tetO-HSP90/hsp904 (light red) cells over a 10, 30 or 60 min $30-42{ }^{\circ} \mathrm{C}$ heat shock.

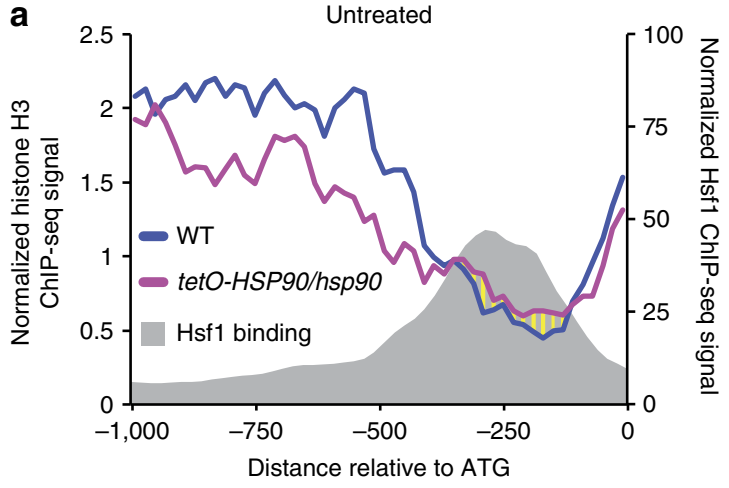

C

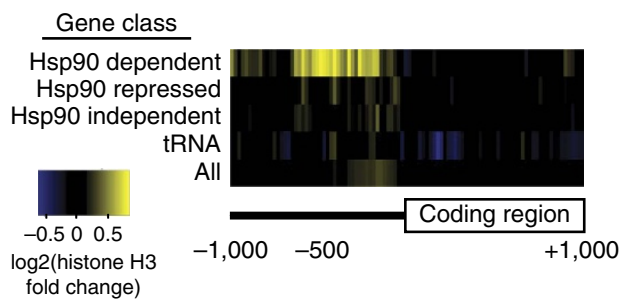

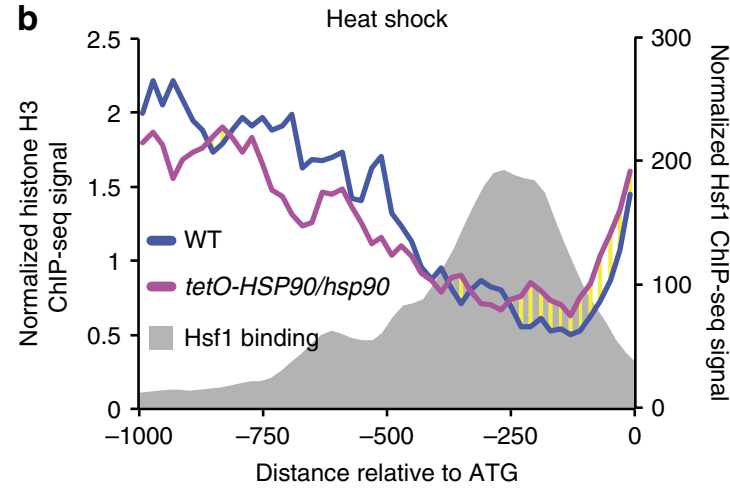

d $\begin{array}{ccc}\square & \square & \square \\ \text { SAGA-regulated } & \text { TFIID-regulated } & \begin{array}{c}\text { SAGA- and TFIID- } \\ \text { regulated }\end{array}\end{array}$

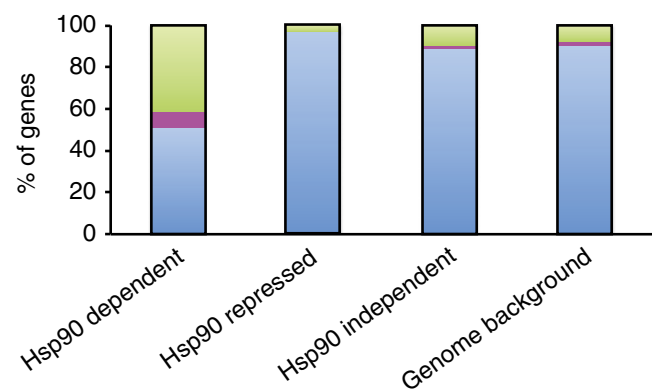

Figure 7 | Hsp90 is required for maintaining nucleosome-free regions. Schematic diagrams showing nucleosome density in wild-type (SN95, blue) and tetO-HSP90/hsp90 (red) cells, as well as Hsf1-TAP-binding position (grey shade) at Hsf1 regulated promoters with (a) and without (b) heat shock. (c) Heat map illustrating nucleosome density changes upon HSP9O depletion $1 \mathrm{~kb}$ upstream and downstream of indicated classes of genes. Yellow represents an increase and blue represents a decrease in nucleosome density. (d) Histogram representing the per cent of Hsp90-dependent or-repressed genes regulated by SAGA or TFIID compared with Hsp90-independent genes and the genome background.

during heat shock impairs histone gene expression. Fourth, by identifying orthologs of $S$. cerevisiae genes controlled by SAGA (Spt-Ada-Gcn5-acetyltransferase) or TFIID (transcription factor II D), we discovered that Hsp90-repressed genes are almost exclusively controlled by TFIID, whereas Hsp90-dependent genes are dominated by SAGA (Fig. 7d); this is in keeping with the 
role of SAGA in regulating stress-induced genes ${ }^{43}$. In both $S$. cerevisiae and $C$. albicans, Hsp90 genetically interacts with SPT3, a component of the SAGA complex ${ }^{38,44}$. SAGA acetylates promoter nucleosomes, which facilitates displacement of nucleosomes ${ }^{45}$; this suggests that nucleosome density may increase at Hsp90 dependent genes due to impaired function of the SAGA complex upon Hsp90 compromise. These mechanisms are not mutually exclusive, and may all contribute to the impact of Hsp90 on transcriptional responses to cellular stress.

\section{Discussion}

The capacity to coordinate sensing and responding to temperature is a driving force for microbial evolution. Beyond enabling adaptation to thermal stress, growth at physiological temperatures is a fundamental requirement for pathogenesis, and mammalian endothermy may have evolved to restrict fungal infections ${ }^{46}$. Our goal was to determine if temperature modulates virulence in a pathogen that has evolved in a relatively stable thermal environment. Using RNA-seq and ChIP-seq, we observed that heat shock induces profound transcriptional remodelling in C. albicans, with upregulation of $\sim 18 \%$ of the genome, which is controlled in large part by Hsfl in concert with Hsp90. Many of the genes induced by heat shock in C. albicans encode HSPs required to re-fold thermally denatured proteins, and components of the ubiquitination/proteolysis pathway that target unfolded proteins for degradation. We also observed that heat shock induces genes involved in filamentation, pathogenesis and adhesion, which were found to be directly regulated by Hsf1 during heat shock (Fig. 1b). This includes the ALS family of genes that are required for adhesion 29,30 , and $R O B 1$, a key regulator of biofilm formation ${ }^{27}$. We found that the transcriptional program induced by a $30-42{ }^{\circ} \mathrm{C}$ heat shock in C. albicans is associated with increased host cell adherence, host cell damage, and virulence (Fig. 4). These findings were validated with a more physiologically relevant heat shock of $37-42^{\circ} \mathrm{C}$; although the magnitude of effects on virulence traits was smaller, it is likely that additional stresses encountered in the human host would amplify the impact of temperature stress. Together, our findings support a model in which Hsf1 has evolved as a key temperature sensor, primed to both protect the cell from thermal insults and enable exploitation of a compromised host.

Motif analysis of Hsfl targets demonstrates that most heat-induced Hsf1 targets, including ALS1, ALS3 and ALS4, contain the motifs GAAnnTTC and TTCn 7 TTC, whereas many constitutively bound Hsfl targets, including HSF1, contain the motif TTCnnGAAnnTTC (Fig. 2e). This suggests that Hsf1 may regulate expression of virulence genes via a distinct mechanism from its regulation of heat shock genes. Contrary to studies in S. cerevisiae $^{47}$, we found that histone binding levels do not change significantly in response to heat shock (Fig. 3c). This suggests three things: first, Hsfl-binding sites are primed for binding; second, upregulation of $H S F 1$ during heat shock drives the increase in Hsfl binding; and third, Hsfl activation does not involve histone eviction. Further, histone levels remain similar at different Hsfl motifs (Supplementary Fig. 4a,b), suggesting that Hsfl-binding sites (and consequently target genes) are predetermined by chromatin architecture. Regulation at this level suggests that Hsfl is able to provide robustness that promotes survival in diverse hostile host niches and rapid cellular responses to signals from the host immune system or microbiota to initiate transitions from commensal to pathogenic states.

The capacity of Hsfl to orchestrate gene expression programs and cellular adaptation is modulated by complex functional relationships with Hsp90. Under basal conditions, depletion of HSP90 leads to the upregulation of a subset of Hsf1 targets
(19 out of 104): those enriched for protein folding and response to heat (Supplementary Table 2). This likely occurs through an increase in HSF1 expression and Hsf1 binding to DNA upon HSP90 depletion (Fig. 6a,b). This fits with the model that Hsp90 represses Hsfl function ${ }^{48-50}$, and our previous observations that depletion of HSP90 induces Hsf1 phosphorylation and upregulates Hsfl targets ${ }^{18}$. In response to heat shock, depletion of HSP90 impairs the rapid global transcriptional response, which could contribute to the reduced virulence of strains depleted of HSP90 in a murine model of candidiasis ${ }^{23}$. The delay may be due in part to the decrease in $H S F 1$ induction resulting in a reduced Hsf1-binding signal during heat shock upon HSP90 depletion (Fig. 6a,c). These effects are likely exacerbated by the increase in promoter nucleosome density observed upon HSP90 depletion at Hsf1 target genes and genes with Hsp90-dependent expression (Fig. 7). This is consistent with findings that Hsp90 enables nucleosome removal from the $S$. cerevisiae GAL1 promoter and recruitment of transcriptional machinery ${ }^{22}$, and suggests a broader role for Hsp90 in nucleosome architecture.

Such alterations in nucleosome architecture likely occur through multiple mechanisms. First, Hsp90 modulates expression of numerous genes that influence nucleosome biology, including the chaperone FPR3, transcription factor $C B F 1$ and histone genes. Second, Hsp90 may influence function of the SAGA complex via interactions with Spt3 in C. albicans ${ }^{44}$. Notably, in S. cerevisiae Hsp90 interacts with multiple components of the SAGA complex, including Ada2, Gcn5, Spt3, Spt4 and Spt15 (ref. 38). The histone acetyltransferase activity of the SAGA complex facilitates promoter nucleosome displacement ${ }^{45}$. Third, given that S. cerevisiae Hsp90 interacts with the chromatin remodelling factors Rvb1/2, Vps1, Swr1 and Aor1 (ref. 38), Hsp90 may modulate chromatin states through interaction with chromatin remodelling factors. Fourth, Hsp90 may regulate nucleosome eviction. Consistent with this possibility, Hsp90 associates with histone H3.1 and the histone chaperone t-NASP in mammals ${ }^{51}$, with depletion of Hsp90 increasing the levels of soluble $\mathrm{H} 3$ and $\mathrm{H} 4$ (ref. 52). The resultant increase in nucleosome occupancy that could arise by these mechanisms would inhibit transcription ${ }^{15}$, with depletion of HSP90 reducing expression of over $75 \%$ of Hsf1 target genes after a $10 \mathrm{~min}$ heat shock. Thus, Hsp90 modulates global transcriptional responses to stress via nucleosome occupancy, and protein homoeostasis via repressive effects on Hsf1.

The mechanisms through which core eukaryotic cellular regulators modulate host-pathogen interactions are only beginning to be understood. C. albicans has evolved elaborate mechanisms to thrive in diverse and hostile environments, where it must resist host defences and mitigate polymicrobial interactions in the highly competitive communities that flourish in commensal and pathogenic states. The coordinated control of the heat shock response with a transcriptional program that promotes pathogenesis suggests that $C$. albicans, as well as other similar pathogens have adapted to febrile temperatures as a ubiquitous host response to infection, exploiting the temperature shift, along with other host responses, as a cue to activate virulence programs. This provides a stunning example of thermal fluctuation as a driving force for host-pathogen co-evolution in the fungal kingdom.

\section{Methods}

Strains and growth conditions. All strains are listed in Supplementary Table 3. Strains were grown in YPD ( $1 \%$ yeast extract, $2 \%$ bactopeptone, $2 \%$ glucose $)^{53}$ To impose an instant heat shock of $30-42^{\circ} \mathrm{C}$, cells were first grown in YPD at $30^{\circ} \mathrm{C}$ to exponential phase, and then half of the volume was removed and mixed with an equal volume of medium that had been pre-warmed to $54^{\circ} \mathrm{C}$ in flasks that had been pre-warmed at $42^{\circ} \mathrm{C}$. To impose an instant heat shock of $37-42^{\circ} \mathrm{C}$, cells were grown in YPD at $37^{\circ} \mathrm{C}$ to exponential phase, and then half of the volume was 
removed and mixed with an equal volume of medium that had been pre-warmed to $47^{\circ} \mathrm{C}$ in flasks that had been pre-warmed at $42^{\circ} \mathrm{C}$. Cells were left at 30 or $37^{\circ} \mathrm{C}$ (untreated controls) or grown at $42^{\circ} \mathrm{C}$ (heat shock) for the times indicated. Doxycycline (BD Biosciences) was added to YPD medium at a concentration of $20 \mu \mathrm{g} \mathrm{ml}^{-1}$ to mediate transcriptional repression from the tet $O$ promoter.

RNA-seq. To comprehensively study the heat shock response, we performed high-throughput sequencing of cDNA (RNA-seq) made from RNA isolated from C. albicans. C. albicans wild-type (CaLC239, SN95) and tetO-HSP90/hsp904 (CaLC1441) cells were grown overnight at $30^{\circ} \mathrm{C}$ in YPD. Stationary phase cultures were split, adjusted to an $\mathrm{OD}_{600}$ of 0.05 in YPD with or without $20 \mu \mathrm{g} \mathrm{ml}{ }^{-1}$ doxycycline (BD Biosciences) and grown for $6 \mathrm{~h}$ to ensure that HSP90 is depleted, but cells are still viable ${ }^{18}$. Cells were then subjected to a heat shock for 10,30 and $60 \mathrm{~min}$, as described in growth conditions. Cells were harvested at 3,000 r.p.m. for $2 \mathrm{~min}$ at $4{ }^{\circ} \mathrm{C}$, washed once with $1 \times \mathrm{PBS}$ and frozen rapidly in liquid nitrogen. RNA was extracted with Triazol (GibcoBRL: Grand Island, NY), as described previously ${ }^{54}$. RNA integrity was assayed on an Agilent Bioanalyser 2,100 (Stockport, UK). TruSeq RNA-seq libraries were prepared according to the manufacturer's instructions (Illumina, Cambridge, UK), and sequenced on the Illumina HiSeq2000 platform. Over $80 \%$ of raw sequences aligned to the C. albicans SC5314 genome sequence (Candida Genome Database) ${ }^{55}$. Gene expression analysis was performed using Partek Genomics Suite software, version 6.6; 2015.

Approximately 20 million reads were generated per sample, over $80 \%$ of which mapped to C. albicans ORFs. DESeq ${ }^{56}$ was used to normalize the data sets and calculate the fold changes and their statistical significance on the basis of three independent biological replicates for each condition (Supplementary Data 5). GOSeq was then used to examine the enrichment of $\mathrm{GO}$ term $\mathrm{s}^{57}$.

ChIP-seq. C. albicans untagged wild-type (CaLC239, SN95) and Hsf1-TAP-tagged (CaLC2993) cells were grown to mid-log phase $\left(\mathrm{OD}_{600} 0.6\right)$ at $30^{\circ} \mathrm{C}$ (untreated) and subjected to a $15 \mathrm{~min}$ heat shock as described in growth conditions. Cells were poured into $50 \mathrm{ml}$ falcon tubes containing $1.2 \mathrm{ml}$ of $37 \%$ formaldehyde up to the $40 \mathrm{ml}$ mark and incubated with gentle rocking for $20 \mathrm{~min}$ at room temperature, after which $10 \mathrm{ml}$ of $2.5 \mathrm{M}$ glycine was added to stop the crosslinking and cells were mixed for $2 \mathrm{~min}$. Cells were harvested at 3,000 r.p.m. for $2 \mathrm{~min}$ at $4{ }^{\circ} \mathrm{C}$, washed twice with ice-cold $1 \times$ TBS and frozen rapidly in liquid nitrogen. Chromatin was extracted as described previously ${ }^{58}$. Briefly, cells were lysed in $500 \mu \mathrm{l}$ of FA lysis buffer $(150 \mathrm{mM} \mathrm{NaCl})$ for $3 \mathrm{~min}$ using a Bullet Blender. Lysis was repeated three times with a $3 \mathrm{~min}$ incubation on ice in between. Chromatin was pelleted by centrifugation at 14,000 r.p.m. for $15 \mathrm{~min}$ at $4{ }^{\circ} \mathrm{C}$ and subsequently re-suspended in $500 \mu \mathrm{l}$ of $\mathrm{FA}$ lysis buffer $(150 \mathrm{mM} \mathrm{NaCl})$. Sonication was used to shear chromatin DNA to around $100-300 \mathrm{bp}$ in size. Chromatin solution was recovered by centrifugation at 14,000 r.p.m. for $30 \mathrm{~min}$ at $4{ }^{\circ} \mathrm{C}$ and kept at $-80{ }^{\circ} \mathrm{C}$ until use. Immuno-precipitation of TAP-tagged Hsf1 was performed by mixing $50 \mu \mathrm{l}$ of chromatin extract with $450 \mu$ l of FA lysis $(150 \mathrm{mM} \mathrm{NaCl})$ and $\sim 15 \mu \mathrm{l}$ packed IgG Sepharose 6 Fast Flow matrix (GE Healthcare) for $3 \mathrm{~h}$ at room temperature on an end-to-end rotator. Similarly, immuno-precipitation of histone $\mathrm{H} 3$ and PolII was executed by first mixing $50 \mu \mathrm{l}$ of chromatin extract with $450 \mu \mathrm{l}$ of FA lysis $(150 \mathrm{mM}$ $\mathrm{NaCl}$ ) and $2 \mu \mathrm{g}$ of anti-H3 (Abcam ab1791) or $2 \mu \mathrm{l}$ of anti-PolII antibodies (Covance 8WG16), respectively, for $1.5 \mathrm{~h}$, followed by incubating the mixture with $\sim 15 \mu \mathrm{l}$ packed Protein A Sepharose (GE Healthcare) for another $1.5 \mathrm{~h}$ at room temperature on an end-to-end rotator. Subsequently, IgG or Protein A Sepharose matrix was transferred to a Corning Costar SpinX centrifuge tube filter and washed according to Fan and colleagues ${ }^{58}$. Immuno-precipitated chromatin DNA was decrosslinked at $65^{\circ} \mathrm{C}$ overnight and was purified using a QIAGEN PCR clean up purification column. Library preparation and multiplex Illumina Sequencing was carried out as described previously ${ }^{59,60}$. Libraries were sequenced by the Illumina HiSeq2500 platform. The nuclear and mitochondrial genome sequences and General Feature File for C. albicans isolate SC5314 v.A21-s02-m08-r01 were downloaded from www.candidagenome.org (GenBank project accession number PRJNA10701). Illumina reads were aligned to the genome using Bowtie with this parameter: $-510-\mathrm{k} 1$, --best ${ }^{61}$.

Identification of Hsf1 targets. Binding sites of Hsf1-TAP were identified using MACS $^{62}$. MACS-called peaks with a $P$ values $<0.05$ were manually inspected and dubious binding sites (peaks with low signal in both untreated and heat conditions and/or portraying a strange shape) were removed from further analysis. Mapping the genes by their ATG position located closest to confident Hsf1-TAP ChIP-seq peaks, identifying direct Hsf1 target genes. A total of 103 genes were identified from 104 MACS peaks from data sets of untreated and heat shock conditions (Supplementary Data 1).

De novo motif discovery. Sequences (200 bp) spanning the summit of the Hsf1-TAP ChIP-seq peaks were subjected to de novo motif analysis using MEME, DREME and HOMER. For extended analysis in identifying additional motifs, peaks with a canonical Hsf1-binding site (GAAnnTTC) 100 bp each side of the summit were removed and the same de novo motif analysis was carried out on the remaining peaks (Supplementary Data 4 ).
Hsf1 binding and nucleosome density (histone H3) analysis. Hsf1 and histone H3 ChIP-seq signals were calculated by counting the number of sequencing read fragments that overlapped within $20 \mathrm{bp}$ sliding windows across a $1 \mathrm{~kb}$ region spanning the summit of each peak or across the $1 \mathrm{~kb}$ promoter and the first $1 \mathrm{~kb}$ coding region of each target gene. The read count was then normalized to the total number of mapped reads in the respective data set. A median was taken for each $20 \mathrm{bp}$ window, and the median (multiplied by a factor of 1,000,000 for $y$ axis scaling for plotting purposes) was used in the ChIP-seq signal plot for the analysed peaks, promoter and coding regions ( $-1,000$ to $+1,000$ with respect to ATG). Nucleosome free regions were systematically identified using the wild-type untreated histone H3 ChIP-seq data by scanning for the lowest Histone H3 signals across $20 \mathrm{bp}$ windows within $500 \mathrm{bp}$ upstream of ATG. If the identified window had zero histone $\mathrm{H} 3$ signal, the first $20 \mathrm{bp}$ neighbouring window with a positive signal is set as the boundary of the nucleosome free region. Histone H3 signals (normalized to total number of mapped reads) at the identified regions were measured and compared between different samples and expressed as $\log _{2}$ fold change. To rule out that the observed effect of HSP 90 depletion (the increased $\mathrm{H} 3$ levels) is not due to a technical artefact, comparative analysis was performed between biological replicates. Two-sample Kolmogorov-Smirnov test was performed between untreated and heat shocked samples (Supplementary Fig. 7d; $P$ values $=7.723 \mathrm{e}-07$ and $5.791 \mathrm{e}-09$ for untreated and heat shock comparisons, respectively).

Pol II ChIP-seq analysis. ChIP-seq against RNA polymerase II (Pol II) was used to determine active transcription activities. Transcription level was measured by the number of ChIP-seq fragments (sequencing reads) across the entire open reading frame of a gene normalized first to the length of the gene followed by the total number of mapped reads of a data set. The normalized number, which is similar to RPKM in the RNA-seq analysis, was used in expression comparisons between untreated and heat shock conditions as well as between wild-type and tetO-HSP90/hsp $90 \Delta$ strains.

Classification of Hsp90 targets. Gene expression (measured by PolII ChIP-seq) before and after heat shock in strains with or without HSP90 depletion was compared. Genes with lower and higher expression upon HSP90 depletion were classified as Hsp90-Dependent (fold change > -2) and Hsp90-Repressed (fold change $>2$ ), respectively, while genes whose expression remain unchanged (fold change between \pm 0.2 to 0 ) are referred to as Hsp90-Independent.

qRT-PCR. To monitor gene expression changes in response to heat shock, strains SN95 (CaLC2993, wild-type) and CaLC2998 (tetO-HSF1/hsf1 1 ) were grown overnight at $30^{\circ} \mathrm{C}$ in YPD. Stationary phase cultures were split, adjusted to an $\mathrm{OD}_{600}$ of $0.2 \mathrm{in} 10 \mathrm{ml}$ YPD with or without doxycycline (BD Biosciences) and cultured at $30^{\circ} \mathrm{C}$ for $16 \mathrm{~h}$. This was necessary to ensure depletion of Hsfl. Cells were diluted once again to an $\mathrm{OD}_{600}$ of 0.2 in the same treatment conditions as the $16 \mathrm{~h}$ and were grown at $30^{\circ} \mathrm{C}$ until mid-log phase $(4 \mathrm{~h})$. Cells were subjected to a $15 \mathrm{~min} 30-42{ }^{\circ} \mathrm{C}$ heat shock as described earlier and $50 \mathrm{ml}$ was harvested from each culture at the specified time, centrifuged at 3,000 r.p.m. for $2 \mathrm{~min}$ at $4{ }^{\circ} \mathrm{C}$, and washed once with $1 \times$ PBS before snap freezing in liquid nitrogen. Pellets were stored at $-80^{\circ} \mathrm{C}$ before extraction. To monitor gene expression upon a $37-42^{\circ} \mathrm{C}$ heat shock, wild-type cells (SN95, CalC239) were grown to mid-log phase in YPD at $37^{\circ} \mathrm{C}$ before heat shock as described earlier. RNA was isolated using the QIAGEN RNeasy kit and cDNA synthesis was performed using the AffinityScript cDNA synthesis kit (Stratagene). PCR was carried out using the SYBR Green JumpStart Taq ReadyMix (Sigma-Aldrich) with the following cycle conditions: $95^{\circ} \mathrm{C}$ for $3 \mathrm{~min}, 95^{\circ} \mathrm{C}$ for $10 \mathrm{~s}$ and $60^{\circ} \mathrm{C}$ for $30 \mathrm{~s}$ for 39 rounds, $95^{\circ} \mathrm{C}$ for $10 \mathrm{~s}, 65^{\circ} \mathrm{C}$ for $5 \mathrm{~s}$. All reactions were done in triplicate using primers listed in Supplementary Table 4. Transcript levels were normalized to ACT1. Data were analysed using the BioRad CFX Manager software, version 3.1 (Biorad).

Heat shock survival assays. To test heat shock survival in different mutants,

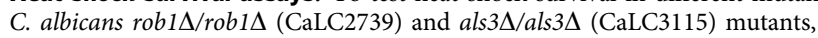
along with their respective wild-type SN152 (CaLC2740) and BWP17 (CaLC3665) (Supplementary Table 3) were grown to mid-log phase in YPD at a starting $\mathrm{OD}_{600}$ of 0.1. Upon reaching mid-log phase, cells were left untreated or were stressed for one hour with a $30-42{ }^{\circ} \mathrm{C}$ heat shock, and CFUs determined by plating onto YPD plates. Viability was calculated as a percentage of survival after a heat shock compared with untreated cells. Experiment was replicated on three separate occasions.

Culture of epithelial cells. The squamous carcinoma of buccal mucosa derived epithelial cell line TR146 was obtained as a gift from Julian Naglik, Kings College London. TR146 cells were routinely grown in DMEM medium containing 10\% heat inactivated FBS (Gibco, 16,000,044) and $\times 1$ antibiotic-antimycotic (Gibco, $15,240,062)$. When TR146 cells were grown in the presence of C. albicans, antibiotic-antimycotic was replaced with $1 \%$ penicillin-streptomycin (Sigma, P4333). Cells were maintained in a humidified incubator at $37^{\circ} \mathrm{C}, 5 \% \mathrm{CO}_{2}$. For adhesion, TR146 oral epithelial cell monolayers were grown to confluence 
in six-well tissue culture plates and serum-starved overnight. For cell damage assays $5 \times 10^{5}$ cells were seeded in 24 -well plates and cultured for $24 \mathrm{~h}$ before experiments.

Cell adherence and damage assays. C. albicans wild-type (SN95, CaLC239) cells were grown to mid-log phase in YPD before subjection to a 15 min heat shock. To determine adhesion, untreated and heat shock treated cells were counted and 100 cells were added to replicate wells containing $1 \mathrm{ml}$ serum-free DMEM for $90 \mathrm{~min}$ at $37^{\circ} \mathrm{C}$ and $5 \% \mathrm{CO}_{2}$ or plated onto replicate control plates. Non-adherent C. albicans cells were removed by aspiration, wells were washed twice with $1 \mathrm{ml}$ PBS, and overlaid with molten Sabouraud's Dextrose Agar (SDA) at $40^{\circ} \mathrm{C}$ (ref. 34). After $24 \mathrm{~h}$ at $30^{\circ} \mathrm{C}$ the resulting colonies were counted and per cent adherence was calculated as (mean adherent CFU/mean total CFU) $\times 100$. To determine cell damage, untreated and heat shock-treated wild-type (SN95, CaLC239) cells with a fungal:epithelial cell multiplicity of infection of 0.01 (ref. 34) were added to TR146 monolayers and incubated at $37^{\circ} \mathrm{C}$ and $5 \% \mathrm{CO}_{2}$ for $20 \mathrm{~h}$. Control wells contained PBS alone. Following incubation, culture supernatant was collected and assayed for LDH using the Cytox 96 Non-Radioactive Cytotoxicity Assay kit (Promega) according to manufacturer's instructions. Replicate LDH measurements were made on the single well, and the assay was repeated on separate days. All data were analysed using a two-tailed t-test with $P<0.05$ considered significant.

Galleria mellonella killing assay. Larvae in their final instar (Next Millennium Farms) were injected with C. albicans wild-type (SN95, CaLC239) cells grown at $30{ }^{\circ} \mathrm{C}$ or subject to a $15 \mathrm{~min} 30-42{ }^{\circ} \mathrm{C}$ heat shock. Briefly, overnight cultures were adjusted to an $\mathrm{OD}_{600} 0.1$ in YPD. Cells were grown to mid-log phase before being subjected to the heat shock. C. albicans cells were washed with PBS and cell densities determined by haemocytometer counts. Dilutions were prepared in PBS and cells were injected at $2.5 \times 10^{5}$ in $10 \mu \mathrm{l}$ into the haemocoel via a distinct proleg, with 20 larvae per infection group. Kill curves were plotted and analysed by the Kaplan-Meier method (GraphPad Prism). Log-rank test was used to determine significance.

Zebrafish care and maintenance. Zebrafish were maintained in re-circulating systems (Aquatic Habitats, Apopka, FL) at the University of Maine Zebrafish Facility with a $14 / 10 \mathrm{~h} \mathrm{light/dark} \mathrm{cycle} \mathrm{and} \mathrm{water} \mathrm{temperature} \mathrm{at} 28^{\circ} \mathrm{C}$. All zebrafish care and husbandry were performed as described previously ${ }^{63}$ All zebrafish care protocols and experiments were performed in accordance with $\mathrm{NIH}$ guidelines under Institutional Animal Care and Use Committee (IACUC) protocol A2012-11- 03.

Wild-type, $A B$, zebrafish larvae were maintained at a density of 150 per dish in $15 \mathrm{~cm}$ petri dishes containing $150 \mathrm{ml}$ of $\mathrm{E} 3$ media $(5 \mathrm{mM} \mathrm{NaCl} ; 0.17 \mathrm{mM} \mathrm{KCl}$; $0.33 \mathrm{mM} \mathrm{CaCl} ; 0.33 \mathrm{mM} \mathrm{MgCl} ; 2 \mathrm{mM}$ HEPES; $\mathrm{pH} 6.8$ ). E3 media was supplemented with $0.3 \mathrm{mgl}^{-1}$ methylene blue for the initial $24 \mathrm{~h}$ to inhibit microbial growth. Larvae were cleaned by changing the E3 media daily. Zebrafish were raised in water containing $15 \mu \mathrm{g} \mathrm{ml}^{-1}$ of 1-phenyl-2-thiourea (PTU) (SigmaAldrich, St Louis, MO) to prevent pigmentation.

Zebrafish hindbrain ventricle infection. C. albicans infection in the hindbrain ventricle was performed as previously described ${ }^{64}$. Briefly, Zebrafish at the prim 25 stage ( $\sim 36 \mathrm{~h}$ post fertilization) were staged according to the method of Kimmel et al. ${ }^{65}$. Before infection larvae were manually dechorionated, and anaesthetized in Tris-buffered tricaine methane sulfonate (tricaine; $200 \mathrm{mg} \mathrm{ml}^{-1}$ ) (Western Chemicals, Inc., Frendale, WA). For each independent experiment, 30 larvae were microinjected with $4 \mathrm{nl}$ of either PBS or $1 \times 10^{7}$ cells per $\mathrm{ml}$ of C. albicans suspension through the otic vesicle into the hindbrain ventricle to achieve a dose of $\sim 15-20$ yeast/larva. Larvae were then screened by microscopy immediately post-infection to ensure correct inoculum sizes and injection location. Data from three independent experiments were plotted. Kill curves were plotted and analysed by the Kaplan-Meier method (GraphPad Prism). Log-rank test was used to determine significance.

Data availability. RNA-sequencing data sets are available at ArrayExpress (www.ebi.ac.uk) under accession code E-MTAB-4075. ChIP-seq data sets are available at the NCBI SRA database (http://www.ncbi.nlm.nih.gov) under accession code SRP071687. The authors declare that all other data supporting the findings of this study are available within the article and its supplementary information files, or from the corresponding author upon request.

\section{References}

1. Kussell, E. \& Leibler, S. Phenotypic diversity, population growth, and information in fluctuating environments. Science 309, 2075-2078 (2005).

2. Tirosh, I., Weinberger, A., Carmi, M. \& Barkai, N. A genetic signature of interspecies variations in gene expression. Nat. Genet. 38, 830-834 (2006).

3. Gasch, A. P. et al. Genomic expression programs in the response of yeast cells to environmental changes. Mol. Biol. Cell 11, 4241-4257 (2000).
4. Underhill, D. M. \& Iliev, I. D. The mycobiota: interactions between commensal fungi and the host immune system. Nat. Rev. Immunol. 14, 405-416 (2014).

5. Odds, F. C. Candida and Candidosis, 2nd edn (Baillere Tindall, Oxford, 1988)

6. Horn, D. L. et al. Epidemiology and outcomes of candidemia in 2019 patients: data from the prospective antifungal therapy alliance registry. Clin. Infect. Dis. 48, 1695-1703 (2009).

7. Shalgi, R. et al. Widespread regulation of translation by elongation pausing in heat shock. Mol. Cell 49, 439-452 (2013).

8. Sorger, P. K., Lewis, M. J. \& Pelham, H. R. Heat shock factor is regulated differently in yeast and HeLa cells. Nature 329, 81-84 (1987).

9. Wiederrecht, G., Shuey, D. J., Kibbe, W. A. \& Parker, C. S. The Saccharomyces and Drosophila heat shock transcription factors are identical in size and DNA binding properties. Cell 48, 507-515 (1987).

10. Nicholls, S., Leach, M. D., Priest, C. L. \& Brown, A. J. Role of the heat shock transcription factor, Hsfl, in a major fungal pathogen that is obligately associated with warm-blooded animals. Mol. Microbiol. 74, 844-861 (2009).

11. Sorger, P. K. \& Pelham, H. R. B. Yeast heat shock factor is an essential DNA-binding protein that exhibits temperature-dependent phosphorylation. Cell 54, 855-864 (1988).

12. Liu, X. D., Liu, P. C. C., Santoro, N. \& Thiele, D. J. Conservation of a stress response: human heat shock transcription factors functionally substitute for yeast HSF. EMBO J. 16, 6466-6477 (1997).

13. Xiao, H., Perisic, O. \& Lis, J. T. Cooperative binding of Drosophila heat shock factor to arrays of a conserved 5 bp unit. Cell 64, 585-593 (1991).

14. Hahn, J. S., Hu, Z., Thiele, D. J. \& Iyer, V. R. Genome-wide analysis of the biology of stress responses through heat shock transcription factor. Mol. Cell. Biol. 24, 5249-5256 (2004).

15. Lee, C.-K., Shibata, Y., Rao, B., Strahl, B. D. \& Lieb, J. D. Evidence for nucleosome depletion at active regulatory regions genome-wide. Nat. Genet. 36, 900-905 (2004)

16. Gross, D. S., English, K. E., Collins, K. W. \& Lee, S. W. Genomic footprinting of the yeast HSP82 promoter reveals marked distortion of the DNA helix and constitutive occupancy of heat shock and TATA elements. J. Mol. Biol. 216, 611-631 (1990).

17. Leach, M. D., Klipp, E., Cowen, L. E. \& Brown, A. J. Fungal Hsp90: a biological transistor that tunes cellular outputs to thermal inputs. Nat. Rev. Microbiol. 10, 693-704 (2012).

18. Leach, M. D. et al. Hsp90 orchestrates transcriptional regulation by Hsfl and cell wall remodelling by MAPK signalling during thermal adaptation in a pathogenic yeast. PLoS Pathog. 8, e1003069 (2012).

19. Nicholls, S. et al. Activation of the heat shock transcription factor Hsf1 is essential for the full virulence of the fungal pathogen Candida albicans. Fungal Genet. Biol. 48, 297-305 (2011).

20. Taipale, M. et al. A quantitative chaperone interaction network reveals the architecture of cellular protein homeostasis pathways. Cell 158, 434-448 (2014).

21. Sawarkar, R., Sievers, C. \& Paro, R. Hsp90 globally targets paused RNA polymerase to regulate gene expression in response to environmental stimuli. Cell 149, 807-818 (2012).

22. Floer, M., Bryant, G. O. \& Ptashne, M. HSP90/70 chaperones are required for rapid nucleosome removal upon induction of the GAL genes of yeast. Proc. Natl Acad. Sci. USA 105, 2975-2980 (2008).

23. Shapiro, R. S. et al. Hsp90 orchestrates temperature-dependent Candida albicans morphogenesis via Ras1-PKA signaling. Curr. Biol. 19, 621-629 (2009).

24. Cowen, L. E. et al. Harnessing Hsp90 function as a powerful, broadly effective therapeutic strategy for fungal infectious disease. Proc. Natl Acad. Sci. USA 106, 2818-2823 (2009).

25. Robbins, N. et al. Hsp90 governs dispersion and drug resistance of fungal biofilms. PLoS Pathog. 7, e1002257 (2011).

26. Murad, A. M. et al. NRG1 represses yeast-hypha morphogenesis and hyphaspecific gene expression in Candida albicans. EMBO J. 20, 4742-4752 (2001).

27. Nobile, C. J. et al. A recently evolved transcriptional network controls biofilm development in Candida albicans. Cell 148, 126-138 (2012).

28. Leach, M. D. \& Cowen, L. E. Membrane fluidity and temperature sensing are coupled via circuitry comprised of Ole1, Rsp5, and Hsf1 in Candida albicans. Eukaryot. Cell 13, 1077-1084 (2014).

29. Fu, Y. et al. Candida albicans Als1p: an adhesin that is a downstream effector of the EFG1 filamentation pathway. Mol. Microbiol. 44, 61-72 (2002).

30. Zhao, X. et al. ALS3 and ALS8 represent a single locus that encodes a Candida albicans adhesin; functional comparisons between Als3p and Als1p. Microbiology 150, 2415-2428 (2004).

31. Trinklein, N. D., Chen, W. C., Kingston, R. E. \& Myers, R. M. Transcriptional regulation and binding of heat shock factor 1 and heat shock factor 2 to 32 human heat shock genes during thermal stress and differentiation. Cell Stress Chaperones 9, 21-28 (2004).

32. Yamamoto, A., Mizukami, Y. \& Sakurai, H. Identification of a novel class of target genes and a novel type of binding sequence of heat shock transcription factor in Saccharomyces cerevisiae. J. Biol. Chem. 280, 11911-11919 (2005). 
33. Schaller, M., Zakikhany, K., Naglik, J. R., Weindl, G. \& Hube, B. Models of oral and vaginal candidiasis based on in vitro reconstituted human epithelia. Nat. Protoc. 1, 2767-2773 (2006).

34. Murciano, C. et al. Evaluation of the role of Candida albicans agglutinin-like sequence (Als) proteins in human oral epithelial cell interactions. PLoS One 7, e33362 (2012).

35. Hoyer, L. L. The ALS gene family of Candida albicans. Trends Microbiol. 9, 176-180 (2001).

36. Phan, Q. T. et al. Als3 is a Candida albicans invasin that binds to cadherins and induces endocytosis by host cells. PLoS Biol. 5, e64 (2007).

37. Leach, M. D., Tyc, K. M., Brown, A. J. P. \& Klipp, E. Modelling the regulation of thermal adaptation in Candida albicans, a major fungal pathogen of humans. PLoS One 7, e32467 (2012).

38. Zhao, R. et al. Navigating the chaperone network: an integrative map of physical and genetic interactions mediated by the Hsp90 chaperone. Cell 120, 715-727 (2005)

39. McClellan, A. J. et al. Diverse cellular functions of the Hsp90 molecular chaperone uncovered using systems approaches. Cell 131, 121-135 (2007).

40. Richmond, T. J. \& Davey, C. A. The structure of DNA in the nucleosome core. Nature 423, 145-150 (2003).

41. Park, S.-K., Xiao, H. \& Lei, M. Nuclear FKBPs, Fpr3 and Fpr4 affect genomewide genes transcription. Mol. Genet. Genomics 289, 125-136 (2014).

42. Zhou, X. \& O'Shea, E. K. Integrated approaches reveal determinants of genome-wide binding and function of the transcription factor Pho4. Mol. Cell 42, 826-836 (2011).

43. Huisinga, K. L. \& Pugh, B. F. A genome-wide housekeeping role for TFIID and a highly regulated stress-related role for SAGA in Saccharomyces cerevisiae. Mol. Cell 13, 573-585 (2004).

44. Diezmann, S., Michaut, M., Shapiro, R. S., Bader, G. D. \& Cowen, L. E. Mapping the Hsp90 genetic interaction network in Candida albicans reveals environmental contingency and rewired circuitry. PLoS Genet. 8, e1002562 (2012).

45. Ringel, A. E., Cieniewicz, A. M., Taverna, S. D. \& Wolberger, C. Nucleosome competition reveals processive acetylation by the SAGA HAT module. Proc. Natl Acad. Sci. USA 112, E5461-E5470 (2015).

46. Bergman, A. \& Casadevall, A. Mammalian endothermy optimally restricts fungi and metabolic costs. mBio 1, e00212-00210 (2010).

47. Zhao, J., Herrera-Diaz, J. \& Gross, D. S. Domain-wide displacement of histones by activated heat shock factor occurs independently of Swi/Snf and is not correlated with RNA polymerase II density. Mol. Cell. Biol. 25, 8985-8999 (2005).

48. Zou, J., Guo, Y., Guettouche, T., Smith, D. F. \& Voellmy, R. Repression of heat shock transcription factor HSF1 activation by HSP90 (HSP90 complex) that forms a stress-sensitive complex with HSF1. Cell 94, 471-480 (1998).

49. Ali, A., Bharadwaj, S., O'Carroll, R. \& Ovsenek, N. HSP90 interacts with and regulates the activity of heat shock factor 1 in Xenopus oocytes. Mol. Cell. Biol. 18, 4949-4960 (1998).

50. Duina, A. A., Kalton, H. M. \& Gaber, R. F. Requirement for Hsp90 and a CyP-40-type cyclophilin in negative regulation of the heat shock response. J. Biol. Chem. 273, 18974-18978 (1998).

51. Campos, E. I. et al. The program for processing newly-synthesized histones H3.1 and H4. Nat. Struct. Mol. Biol. 17, 1343-1351 (2010).

52. Cook, Adam J. L., Gurard-Levin, Zachary, A., Vassias, I. \& Almouzni, G. A specific function for the histone chaperone NASP to fine-tune a reservoir of soluble H3-H4 in the histone supply chain. Mol. Cell 44, 918-927 (2011)

53. Sherman, F. Getting started with yeast. Methods Enzymol. 194, 3-21 (1991).

54. Hauser, N. C. et al. Transcriptional profiling on all open reading frames of Saccharomyces cerevisiae. Yeast 14, 1209-1221 (1998).

55. Inglis, D. O. et al. The Candida genome database incorporates multiple Candida species: multispecies search and analysis tools with curated gene and protein information for Candida albicans and Candida glabrata. Nucleic Acids Res. 40, D667-D674 (2012)

56. Anders, S. \& Huber, W. Differential expression analysis for sequence count data. Genome Biol. 11, R106-R106 (2010)

57. Young, M. D., Wakefield, M. J., Smyth, G. K. \& Oshlack, A. Gene ontology analysis for RNA-seq: accounting for selection bias. Genome Biol. 11, R14-R14 (2010).

58. Fan, X., Lamarre-Vincent, N., Wang, Q. \& Struhl, K. Extensive chromatin fragmentation improves enrichment of protein binding sites in chromatin immunoprecipitation experiments. Nucleic Acids Res. 36, e125-e125 (2008).
59. Wong, K. H. \& Struhl, K. The Cyc8-Tup1 complex inhibits transcription primarily by masking the activation domain of the recruiting protein. Genes Dev. 25, 2525-2539 (2011)

60. Wong, K. H., Jin, Y. \& Moqtaderi, Z. in Current Protocols in Molecular Biology Ch. 7John Wiley \& Sons, Inc., 2013).

61. Langmead, B., Trapnell, C., Pop, M. \& Salzberg, S. L. Ultrafast and memory-efficient alignment of short DNA sequences to the human genome. Genome Biology 10, 1-10 (2009).

62. Zhang, Y. et al. Model-based analysis of ChIP-Seq (MACS). Genome Biol. 9, R137-R137 (2008).

63. Westerfield, M. A Guide for the Laboratory Use of Zebrafish (Danio rerio) (University of Oregon Press, 2000).

64. Brothers, K. M., Newman, Z. R. \& Wheeler, R. T. Live imaging of disseminated candidiasis in zebrafish reveals role of phagocyte oxidase in limiting filamentous growth. Eukaryot. Cell 10, 932-944 (2011).

65. Kimmel, C. B., Ballard, W. W., Kimmel, S. R., Ullmann, B. \& Schilling, T. F. Stages of embryonic development of the zebrafish. Dev. Dyn. 203, 253-310 (1995).

\section{Acknowledgements}

We thank Karim Gharbi and Urmi Trivedi for their assistance with RNA sequencing, carried out in the GenePool genomics facility (University of Edinburgh). We also thank Susan Fairley and Eduardo De Paiva Alves (Centre for Genome Enabled Biology and Medicine, University of Aberdeen) for help with the initial bioinformatics analysis. We thank Aaron Mitchell for kindly providing the ALS3 mutant, Julian Naglik for the gift of TR146 cells, and Jon Richardson for technical assistance. We thank the Genomics and Bioinformatics core of the Faculty of Health Sciences for Next Generation Sequencing and Bioinformatics support, the Information and Communication Technology Office a the University of Macau for providing access to a High Performance Computer and Jacky Chan and William Pang for their expert support on the High Performance Computer. Finally, we thank Amanda Veri for generating CaLC2928. M.D.L. is supported by a Sir Henry Wellcome Postdoctoral Fellowship (Wellcome Trust 096072), R.A.F. by a Wellcome Trust-Massachusetts Institute of Technology (MIT) Postdoctoral Fellowship, L.E.C. by a Canada Research Chair in Microbial Genomics and Infectious Disease and by Canadian Institutes of Health Research Grants MOP-119520 and MOP-86452, A.J. P.B. was supported by the UK Biotechnology and Biological Sciences Research Council (BB/ F00513X/1) and by the European Research Council (ERC-2009-AdG-249793-STRIFE), KHW is supported by the Science and Technology Development Fund of Macau S.A.R (FDCT) (085/2014/A2) and the Research and Development Administrative Office of the University of Macau (SRG2014-00003-FHS) and R.T.W. by the Burroughs Wellcome fund and NIH R15AO094406.

\section{Author contributions}

M.D.L. conceived the project. M.D.L., K.H.W., K.T., Z.M., R.A.F., C.A.C., A.J.P.B. and L.E.C. designed and interpreted RNA-seq and ChIP-seq experiments. M.D.L. designed and performed experiments in tissue culture and G. mellonella. L.A.W. and R.T.W designed and performed zebrafish virulence assays. M.D.L., K.H.W. and L.E.C. wrote the manuscript. All authors contributed to preparation of the manuscript.

\section{Additional information}

Supplementary Information accompanies this paper at http://www.nature.com/ naturecommunication

Competing financial interests: The authors declare no competing financial interests.

Reprints and permission information is available online at http://npg.nature.com/ reprintsandpermissions/

How to cite this article: Leach, M. D. et al. Hsfl and Hsp90 orchestrate temperaturedependent global transcriptional remodelling and chromatin architecture in Candida albicans. Nat. Commun. 7:11704 doi: 10.1038/ncomms11704 (2016).

This work is licensed under a Creative Commons Attribution 4.0 International License. The images or other third party material in this article are included in the article's Creative Commons license, unless indicated otherwise in the credit line; if the material is not included under the Creative Commons license, users will need to obtain permission from the license holder to reproduce the material. To view a copy of this license, visit http://creativecommons.org/licenses/by/4.0/ 